Supplement of Geosci. Model Dev., 10, 1175-1197, 2017

http://www.geosci-model-dev.net/10/1175/2017/

doi:10.5194/gmd-10-1175-2017-supplement

(C) Author(s) 2017. CC Attribution 3.0 License.

(c) (i)

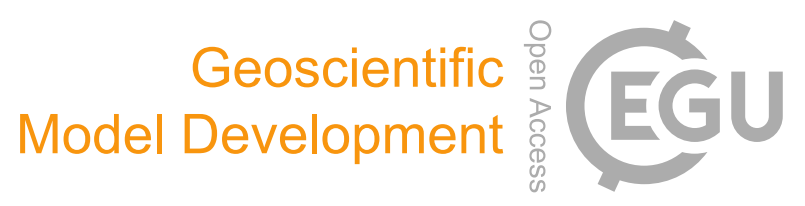

Supplement of

\title{
The Fire Modeling Intercomparison Project (FireMIP), phase 1: experimental and analytical protocols with detailed model descriptions
}

Sam S. Rabin et al.

Correspondence to: Sam S. Rabin (sam.rabin@kit.edu)

The copyright of individual parts of the supplement might differ from the CC-BY 3.0 licence. 


\section{The Fire Modeling Intercomparison Project (FireMIP), phase 1: Experimental and analytical protocols Supplementary tables describing fire models}

\section{List of symbols}

$\alpha_{m}$ Per-capita ignition frequency (ignitions person ${ }^{-1}$ month $^{-1}$ ). See Table ST]. I], B4

$\beta$ Packing ratio (unitless). See Table $5 \mathrm{SW}$. 21]

$\beta_{\text {op }}$ Optimal packing ratio (unitless). See Table STI . 2]

$\Gamma^{\prime}$ Optimal reaction velocity $\left(\mathrm{min}^{-1}\right)$. See Table $\mathbf{S T}$. [1, 2], 25]

$10 \Gamma_{E}^{\prime}$ Maximum reaction velocity as used by MC-Fire for energy release equations $\left(\mathrm{min}^{-1}\right)$. See Table ST]. [1, [1] $\Gamma_{E, \max }^{\prime}$ Maximum reaction velocity as used by MC-Fire for energy release equations $\left(\mathrm{min}^{-1}\right)$. See Table STI 20 $\Gamma_{\text {max }}^{\prime}$ Maximum reaction velocity $\left(\min ^{-1}\right)$. See Table $\mathbf{S W}$. $2 \mathbb{1}$

$\Gamma_{R}^{\prime}$ Maximum reaction velocity as used by MC-Fire for rate of spread equations $\left(\mathrm{min}^{-1}\right)$. See Table [S], [1], 2]

$\Gamma_{R, \max }^{\prime}$ Maximum reaction velocity as used by MC-Fire for rate of spread equations $\left(\mathrm{min}^{-1}\right)$. See Table $\mathbf{S W}$. $2 \mathbb{J}$

$15 \epsilon$ Effective heating number (unitless). See Table $\mathbf{5 ]}$. [15, 2]

$\eta_{M}$ Moisture damping coefficient (unitless, range [0,1]). See Table [S]l. 21], 25]

$\eta_{M, d}$ Moisture damping coefficient for dead fuels (unitless, range [0,1]). See Table ST]. 2$]$

$\eta_{M, l}$ Moisture damping coefficient for live fuels (unitless, range [0,1]). See Table $\mathbf{S T}$. 2]

$\eta_{S}$ Mineral damping coefficient (unitless, range [0,1]). See Table 58. [8, 211, 25]

$20 \theta$ Soil moisture (unitless, range [0,1]). 14, 22

$\theta_{1}$ Soil moisture in uppermost soil layer (unitless, range [0,1]). 目, ㄸ4- [6, 미

$\theta_{5 \mathrm{~cm}}$ Soil moisture in uppermost $5 \mathrm{~cm}$ of soil (unitless, range [0,1]). [4]

$\theta_{e}$ Soil moisture of extinction (unitless, range [0,1]). [4, 30]

$\theta_{m m}$ Plant-available soil moisture (mm). 10

$\theta_{\text {root }}$ Soil moisture in rooting zone (unitless, range [0,1]). [4, [5]

$\mu_{S}$ Mineral content fraction of dead fuels (0.055). See Table 5$]$. [2], 223]

$\xi$ Propagating flux ratio (unitless, range [0,1]). See Table [S]l. [2, [15, 2]1]

$\rho_{b}$ Fuel bulk density $\left(\mathrm{kg} \mathrm{C} \mathrm{m}^{-3}\right)$. See Table $\mathrm{ST}$. [15, [2], [28, [30, [3]

$\rho_{b, l g}$ Fuel bulk density of live grass $\left(\mathrm{kg} \mathrm{C} \mathrm{m}^{-3}\right)$. See Table ST6. 15, 26

$30 \rho_{p}$ Oven-dry particle density $\left(\mathrm{kg} \mathrm{C} \mathrm{m}^{-3}\right)$. See Table $\mathbf{5 8}$. [18, 201] 


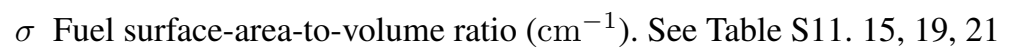

$\sigma_{d, 1 h}$ Fuel surface-area-to-volume ratio: 1-hour dead fuels $\left(\mathrm{cm}^{-1}\right)$. [15, 미, 21, [32

$\sigma_{d, 10 h}$ Fuel surface-area-to-volume ratio: 10-hour dead fuels $\left(\mathrm{cm}^{-1}\right)$. 15, प19, 201

$\sigma_{d, 100 h}$ Fuel surface-area-to-volume ratio: 100-hour dead fuels $\left(\mathrm{cm}^{-1}\right)$. [5, [19, 201]

$5 \sigma_{d, 1000 h}$ Fuel surface-area-to-volume ratio: 1000-hour dead fuels $\left(\mathrm{cm}^{-1}\right)$. ㄸ5 , 2]

$\sigma_{E}$ Fuel surface-area-to-volume ratio $\left(\mathrm{cm}^{-1}\right)$ as used by MC-Fire for energy release equations. See Table $\mathbf{S W}$. 2], 25]

$\sigma_{l, h}$ Fuel surface-area-to-volume ratio: Live herbaceous vegetation $\left(\mathrm{cm}^{-1}\right)$. See Table [27]. 2], 132]

$\sigma_{l, s}$ Fuel surface-area-to-volume ratio: Live shrubby vegetation $\left(\mathrm{cm}^{-1}\right)$. See Table [27]. 2], [3]

$\sigma_{R}$ Fuel surface-area-to-volume ratio $\left(\mathrm{cm}^{-1}\right)$ as used by MC-Fire for rate of spread equations. See Table $\mathbf{S T}$. $2 \mathbb{D}$

$10 \tau$ Fire residence time (min). See Table S15. 12, 211, 25]

$\tau_{*}$ Critical fire residence time (min). See Table [515. 25]

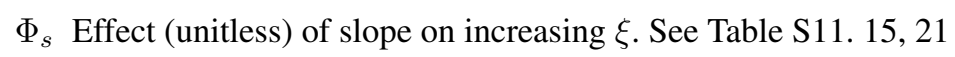

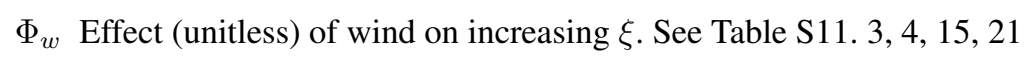

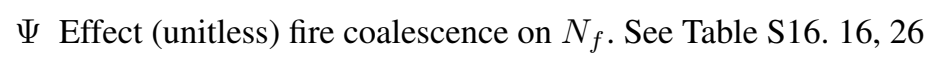

$15 \omega$ Fuel moisture (unitless). See Table \$59. [15, 미, 201]

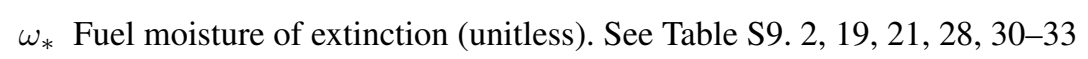

$\omega_{d, 1 h}$ Fuel moisture: Dead 1-hour fuels (unitless). See Table \$59. [2, [1, 211, 23]

$\omega_{d, 1 h / *}$ Fuel moisture of dead 1-hour fuels relative to moisture of extinction (unitless): $\frac{\omega_{d, 1 h}}{\omega_{a \rightarrow}} \cdot[23]$

$\omega_{d, 100 h}$ Fuel moisture: Dead 100-hour fuels (unitless). See Table \$59. [1, [1, 23], 26

$20 \omega_{d, 1000 h}$ Fuel moisture: Dead 1000-hour fuels (unitless). See Table \$9. [3, 192, 223, 26

$\omega_{d, 10 h}$ Fuel moisture: Dead 10-hour fuels (unitless). See Table \$S9. [9, [2]

$\omega_{\text {duff }}$ Fuel moisture: Duff (unitless). See Table 59. [9, 23]

$\omega_{f f}$ Combined fuel moisture: Fine fuels (i.e., live grass and dead 1-hour fuels; unitless). See Table [59. ए, ए9

$\omega_{f f / *}$ Combined fuel moisture of fine fuels relative to moisture of extinction (unitless): $\frac{\omega_{f t}}{w_{\mathbb{Z}}} \cdot[23$

$25 \omega_{l}$ Surface-area-weighted moisture of live fuels (unitless). See Table 59. [19, 20]

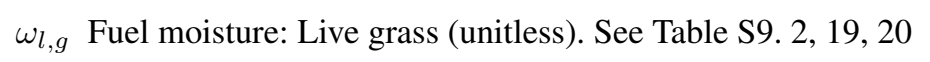

$\omega_{l, g / *}$ Fuel moisture of live grass relative to moisture of extinction (unitless): $\frac{\omega_{l, g}}{[\omega]}$. [23]

$\omega_{l, s}$ Fuel moisture: Live shrubby vegetation (unitless). See Table \$9. [प, 25]

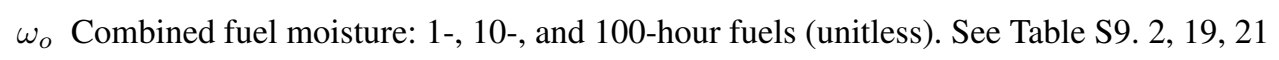

$30 \omega_{o / *}$ Combined fuel moisture of 1-, 10-, and 100-hour fuels relative to moisture of extinction (unitless): $\frac{\omega_{a}}{\omega_{\mathbb{2}}}$. [14, , 211, 223 
$\omega_{x / *}$ General fuel moisture relative to moisture of extinction (unitless). See Table \$9. 4, 매, 미

$\widehat{a}$ Used by LPJ-GUESS-BLAZE to compute burned area. See Table \$223. [0, 229

$A$ Used to compute $[$. See Table $\mathrm{ST}$. 2]

$A_{c}$ Average contiguous non-cropland area $\left(\mathrm{km}^{2}\right)$. प5

$5 A_{E}$ Used to compute $\Gamma_{E}^{\prime}$. See Table [1]. 2]

$A_{g}$ Area of grid cell $\left(\mathrm{km}^{2}\right)$. Only includes land on which the described fire model runs. For example, if the fire model excludes

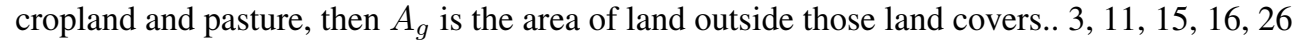

$A_{R}$ Used to compute $\Gamma_{B}^{\prime}$. See Table $\mathbf{S W}$. 2]

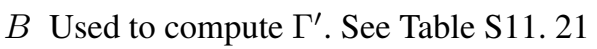

$10 B A_{y}$ Total gridcell burned area so far this year $\left(\mathrm{km}^{2}\right)$. 메, 따

$B A$ Total gridcell burned area $\left(\mathrm{km}^{2}\right.$ timestep $\left.{ }^{-1}\right)$, excluding special fire types described in Table $\mathbf{5 3}$. [16, [26

$B A_{20}$ Mean annual gridcell burned area over the past 20 years $\left(\mathrm{km}^{2}\right)$.

$B A_{p f}$ Burned area per fire $\left(\mathrm{km}^{2}\right)$, accounting for suppressive effects of population density (S $S_{P D, b a}$ or $\left.\mathrm{g}\right)$, GDP (SSDP,ba] $)$,

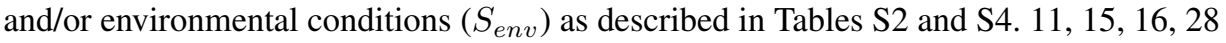

$15 B A_{p f, \text { uns }}$ Unsuppressed burned area per fire $\left(\mathrm{km}^{2}\right)$. Does not consider suppressive effects of population density $\left(S_{P D, b a}\right.$ or

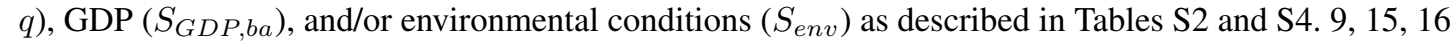

$b_{n d, 100 h}$ Used by MC-Fire to calculate $\omega_{d, 100 h}$. See Table $\mathbf{S 1 6}$. [19, [26

${ }_{b n d} d_{d, 1000 h}$ Used by MC-Fire to calculate $\omega_{d, 1000 h}$. See Table $\mathbf{S 1 6}$. 26

BT Bark thickness (cm). See Table S15. [1, [25, [30, [3]

20 BUI Fuel build-up index (van Wagner and Pickett, 1985; van Wagner, 1987). See Table 516. 15, 26

$\widehat{B U I_{*}}$ PFT-specific threshold build-up index (unitless). See Table 527 . [15, (32]

$C$ Used to compute $\Phi_{\psi}$. See Table $\mathrm{ST}$. 21]

$C F$ Fraction of crown in flaming zone (unitless). See Table [5]5. [1, [23, [25, [28, [30, [32, [3]

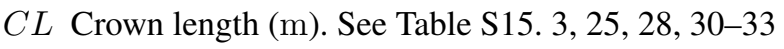

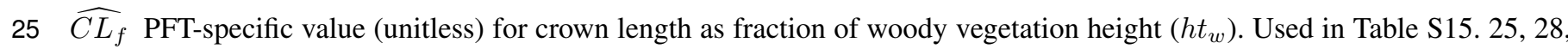
[30- B3]

$\mathbb{Z}_{1000 h}$ Drying parameter $\left({ }^{\circ} \mathrm{C}^{-2}\right)$ : 1000-hour fuels. See Table $\mathbf{S T 0}$. 20]

$\mathbb{Z}_{100 h}$ Drying parameter $\left({ }^{\circ} \mathrm{C}^{-2}\right)$ : 100-hour fuels. See Table $\mathbf{S T 0}$. 20.

$\mathbb{Z}_{l, g}$ Drying parameter $\left({ }^{\circ} \mathrm{C}^{-2}\right)$ : Live grass. See Table $\mathbf{S T 0}$. [20

30

$\mathbb{\varpi}_{10 h}$ Drying parameter $\left({ }^{\circ} \mathrm{C}^{-2}\right)$ : 10-hour fuels. See Table $\mathbf{5 ] 0}$. 20 
$d$ Drying parameter $\left({ }^{\circ} \mathrm{C}^{-2}\right)$. 3, 目, 世1, 20

$\Xi_{1 h}$ Drying parameter $\left({ }^{\circ} \mathrm{C}^{-2}\right)$ : 1-hour fuels. See Table $\mathbf{S T 0}$. [20]

$\widehat{D_{1}}$ Parameter used by LPJ-LMfire for calculating DBH $\left(\mathrm{cm} \mathrm{m}^{-1}\right)$. See Table $\mathbf{5 2 6}$. [25, [1]

$\widehat{D_{2}}$ Parameter used by LPJ-LMfire for calculating DBH $(\mathrm{cm})$. See Table $\mathbf{5 2 6}$. 25, [1]

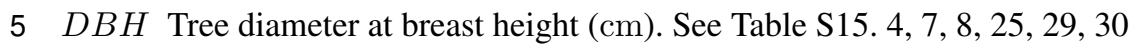

depth Fuel bed depth (ft). See Table \$16. [, 미, 21, 26

DF Drought factor used by LPJ-GUESS-BLAZE to compute FDIMcA. See Table ST6. 26

$\widehat{D F F}_{1 h}$ Dead fuel fraction (unitless): 1-hour fuels. Used by MC-Fire; see Tables [57, 527. 기, 132

$\widehat{D F F}_{10 h}$ Dead fuel fraction (unitless): 10-hour fuels. Used by MC-Fire; see Tables [57, [27]. [17, [32]

$10 \widehat{D F F}_{100 h}$ Dead fuel fraction (unitless): 100-hour fuels. Used by MC-Fire; see Tables S7, S27. [17, [32]

$\widehat{D F F}_{1000 h}$ Dead fuel fraction (unitless): 1000-hour fuels. Used by MC-Fire; see Tables 57, [527. [17, [32]

$\widehat{D R}$ PFT-specific ratio (unitless) of “decomposable" to "resistant” litter. Used by JULES-INFERNO; see Table [S21. [4], 288

$E$ Used to compute $\Phi_{\nu}$. See Table $\mathbf{S W}$. 2]

$e^{*}$ Saturation water vapor pressure (hPa), after Goft and Gratch (1946). [14

$15 e m c_{c}$ Corrected equilibrium moisture content (unitless). See Table ST6. 26

$e m c_{\text {coarse,min }}$ Minimum equilibrium moisture content, coarse fuels (unitless). See Table \$]6. [प, [26

$e m c_{u}$ Uncorrected equilibrium moisture content (unitless). See Table 5T6. [26

$e m c_{u, \max }$ Uncorrected equilibrium moisture content: daily maximum (unitless). See Table S16. 26

$e m c_{u, \min }$ Uncorrected equilibrium moisture content: daily minimum (unitless). See Table $\mathbf{5 T 6}$. 26

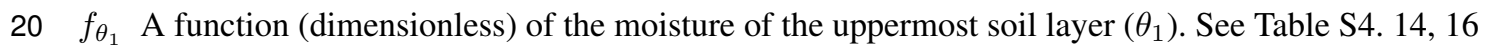

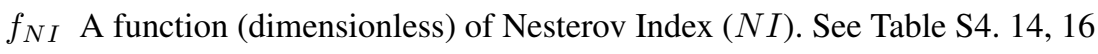

$f_{\mathbb{P D}}$ A function (dimensionless) of population density (PD). See Table $\mathbf{5 2}$. [2, 미

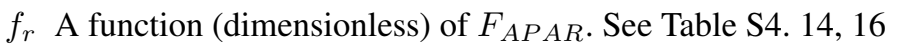

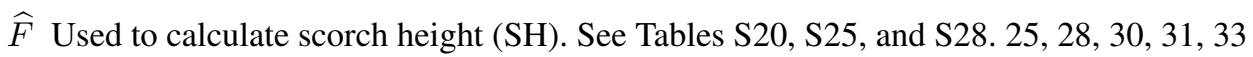

$25 F A_{w, C a}$ Fraction of woody vegetation affected by cambial scorch. See Table S15. 25, [32]

$F A_{w, C a+C r}$ Fraction of woody vegetation affected by crown scorch OR cambial scorch. See Table [15. [22, 221, 25]

$F A_{w, C r}$ Fraction of woody vegetation affected by crown scorch. See Table $\mathbf{5 1 5}$. 18, 223, 25, [32]

$F_{A P A R}$ Fraction absorbed of photosynthetically active radiation (unitless). 目, [4]

$F_{C 2 G}$ Daily cloud-to-ground lightning flash rate $\left(\mathrm{km}^{-2} \mathrm{~d}^{-1}\right)$. س 
$F_{C 2 G, y r}$ Annual cloud-to-ground lightning flash rate $\left(\mathrm{km}^{-2} \mathrm{y}^{-1}\right)$. Шा

$F_{\text {total }}$ Daily total (cloud-to-ground plus within-cloud) lightning flash rate $\left(\mathrm{km}^{-2} \mathrm{~d}^{-1}\right)$, calibrated according to LIS/OTD lightning flash rate climatology. Used by ORCHIDEE-SPITFIRE; see model description paragraph and Table ST. WI

$F C_{d, 1 h}$ Fractional combustion: Dead 1-hour fuels. See Tables ST2 and [S]3. [2]

$5 F C_{d, 10 h}$ Fractional combustion: Dead 10-hour fuels. See Tables $\mathbf{S T 2}$ and $\left.\mathbf{S T 3} .23\right]$

$F C_{d, 100 h}$ Fractional combustion: Dead 100-hour fuels. See Tables [5]2 and [5]3]. [23]

$F C_{d, 1000 h}$ Fractional combustion: Dead 1000-hour fuels. See Tables $\$[2]$ and $\mathbf{S 1 3}$. [23]

$\widehat{F C}_{d, 1000 h, \max }$ PFT-specific maximum fractional combustion: Dead 1000-hour fuels. Used by ORCHIDEE-SPITFIRE; see Table [528, [23, [3]

$10 \widehat{F C}_{d, 100 h, \max }$ PFT-specific maximum fractional combustion: Dead 100-hour fuels. Used by ORCHIDEE-SPITFIRE; see Table 528.231, [3]

$F C_{d, \text { root }}$ Fractional combustion: Fine root litter. See Tables ST2 and [5]3]. 22]

$F C_{d, l e a f}$ Fractional combustion: Leaf litter. See Table \$12. 22

$F C_{d, l i t t e r}$ Fractional combustion: All litter. See Table S12. 22, 27, 28]

$15 F C_{d, \text { stem }}$ Fractional combustion: Stem litter. See Table $\mathbf{5 1 2}[2]$

$F C_{l, 1 h}$ Fractional combustion: Live 1-hour fuels. Note that this applies only to woody PFTs, not grasses. See Table [5]3]. [23, 25]

$F C_{l, 10 h}$ Fractional combustion: Live 10-hour fuels. See Table [5]31. 223, 25]

$F C_{l, 100 h}$ Fractional combustion: Live 100-hour fuels. See Table S[3]. 223]

$20 F C_{l, 1000 h}$ Fractional combustion: Live 1000-hour fuels. See Table ST31. [23]

$F C_{l, g r a s s}$ Fractional combustion: Live grass. See Tables $\mathbf{S T 2}$ and $\mathbf{S T 3}$. [22, 223

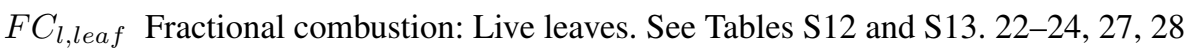

$\widehat{F C}_{l, l e a f, \text { max }}$ PFT-specific maximum fractional combustion: Live leaves. Used by JULES-INFERNO. See Table \$271. [2]

$\widehat{F C}_{l, l \text { eaf,min }}$ PFT-specific minimum fractional combustion: Live leaves. Used by JULES-INFERNO. See Table \$2]. 22, 228

$25 F C_{l, \text { root }}$ Fractional combustion: Live fine roots. See Tables $\mathrm{SI2}$ and $\mathrm{SI3}$. 22

$F C_{l, \text { stem }}$ Fractional combustion: Live stems. See Tables 512 and $\mathbf{5 1 3}$. 222-24, 27, 28

$\widehat{F C}_{l, \text { stem,max }}$ PFT-specific maximum fractional combustion: Live stems. Used by JULES-INFERNO. See Table \$21]. [22, [28]

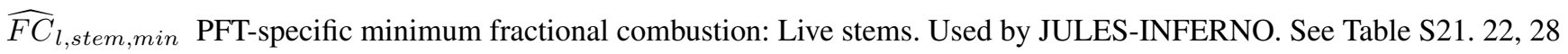

$F C_{l, t s}$ Fractional combustion: Live transfer and storage carbon. See Table [5]7. [2]

30 FDI Fire Danger Index (unitless). See Table \$4. 메, 나-16

$F D I_{M c A}$ McArthur Fire Danger Index (unitless, Noble et al, 1980). See Table [5]6. 4, [15, 26 
FFMC Fine fuel moisture code (unitless). See Table 5]6. [5, 26

$\widehat{F F M C}$ * PFT-specific threshold fine fuel moisture code (unitless). See Table \$27. [5]

$F K_{w}$ Total fraction killed, woody PFTs only. See Table $\mathbf{5 1 5}$. 25]

FK $K_{\text {grass }}$ Fraction killed: Grass. See Table [5]5. [25]

$5 \quad F K_{\text {leaf }}$ Fraction killed: Leaves. See Table S14. [24, 27, [28]

$F K_{w, 1 h}$ Fraction killed: Live 1-hour fuels. Applies to woody PFTs only. See Table [515. 25

$F K_{w, 10 h}$ Fraction killed: Live 10-hour fuels. Applies to woody PFTs only. See Table [S15. 25$]$

$F K_{w, 100 h}$ Fraction killed: Live 100-hour fuels. Applies to woody PFTs only. See Table [515. 25$]$

$F K_{w, 1000 h}$ Fraction killed: Live 1000-hour fuels. Applies to woody PFTs only. See Table [515. 25$]$

$10 F K_{\text {root }}$ Fraction killed: Roots. See Table [S14. 24, 27, [28]

FK $K_{\text {stem }}$ Fraction killed: Stems. See Table 514. 24, 27, 28

$F K_{S t o H}$ Fraction of sapwood carbon transferred to heartwood (i.e., from the live portion of the stem to the dead portion) due to fire, after previous combustion and mortality factors are applied. See Table 5]7. 24, 27]

FK tree Total fraction killed: Trees. See Table S15, 25, 20

$15 F K_{t s}$ Fraction killed: Transfer and storage C. See Table [5]7. 27]

FLA Fuel loading adjustment (unitless). See Table \$1]6. [1, 21], 26

$\widehat{F R I}_{\max }$ Maximum fire return interval (yr). Used by MC-Fire; see Table [27. [15, [32]

$\widehat{F R I}_{\text {min }}$ PFT-specific minimum fire return interval (yr). Used by MC-Fire; see Table [27]. [15, [32]

$G_{a g}$ Fraction of biomass that is aboveground. Additions to subscripts can specify pool-specific fractions.. [4, [प], 222, 231, 22]

$20 G_{b g}$ Fraction of biomass that is belowground. Additions to subscripts can specify pool-specific fractions.. [77, 20]

$G D P_{p c}$ Gross domestic product per capita (2005 US\$ person ${ }^{-1}$ ). Q, 미

$g_{W}$ Reduction (unitless, range [0,1]) of forward rate of spread ([ROS7) based on wind speed. See Table [55. [5]

$h$ Fuel heat content $\left(\mathrm{kJ} \mathrm{kg}^{-1}\right)$. See Table 58 . 18, 132

$H B$ Ellipse head-to-back ratio (unitless). See Table [55. [5]

$25 H_{h}$ Fraction of gridcell that is herbaceous (unitless). 표, ㅁ

$h t_{w}$ Height of woody vegetation (m). See Table S15. [1, 25, 29, 32, [3]

$H_{w}$ Fraction of gridcell that is woody (unitless). [5]

$I_{A}$ Anthropogenic ignition rate (ignitions $\mathrm{km}^{-2} \mathrm{day}^{-1}$ ). See Table [S]. Does not consider suppressive effects of population density $\left(S_{P D, n \rrbracket}\right)$ or GDP $\left(S_{G D P, n \rrbracket}\right)$ as described in Table $\mathbf{5 2}$, nor of environmental conditions $\left(S_{e n v, n \rrbracket}\right)$ as described in Table S4. 피, 16 
$I_{L}$ Lightning ignition rate (ignitions $\mathrm{km}^{-2} \mathrm{day}^{-1}$ ). See Table $\mathbf{S T}$. Does not consider suppressive effects of population density $\left(S_{P D, n}\right)$ or GDP $\left(S_{G D P, n}\right)$ as described in Table $\sqrt{S 2}$, nor of environmental conditions $\left(S_{e n v, n}\right)$ as described in Table S4. 매, ए16

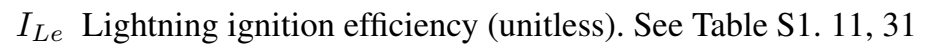

$5 \quad I_{R}$ Reaction intensity $\left(\mathrm{kJ} \mathrm{m}^{-2} \mathrm{~min}^{-1}\right)$. See Table $\mathrm{ST}$. [15, 2]

$I_{R, E}$ Reaction intensity as used by MC-Fire for energy release equations $\left(\mathrm{kJ} \mathrm{m}^{-2} \mathrm{~min}^{-1}\right)$. See Table $\mathbf{S W}$. 201]

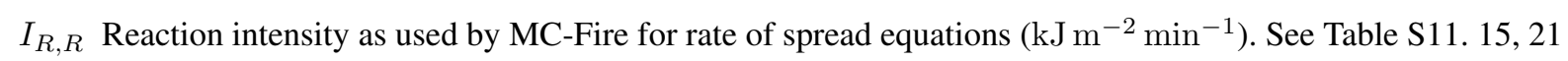

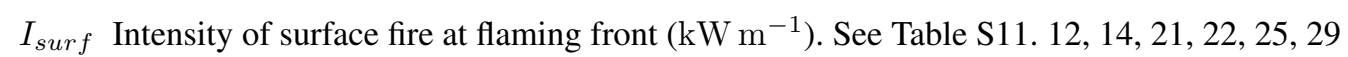

$\widehat{k_{2}}$ Used for calculating tree diameter at breast height (DBH). See Table [525. [25, [30]

$\widehat{k_{3}}$ Used for calculating tree diameter at breast height (DBH). See Table [525. [25, [30]

KBDI Keetch-Byram Drought Index (Keetch and Byram, 1968). 26

$L B$ Ellipse length-to-breadth ratio (unitless). See Table [55. [5]

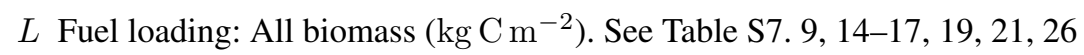

$L_{b, g}$ Fuel loading: Brown grass $\left(\mathrm{kg} \mathrm{C} \mathrm{m}^{-2}\right)$. Used by CTEM as an intermediate pool between grass turnover and the litter pool

$\widehat{L D}$ PFT-specific fuel bed load-to-depth ratio $\left(\mathrm{ft}\left(\mathrm{T} \mathrm{DM} \mathrm{ac}^{-1}\right)^{-1}\right)$. Used by MC-Fire to calculate depth; see Table $\mathbf{5 2 7}$. 26, B2

$L_{d}$ Fuel loading: Dead biomass ( $\mathrm{kg} \mathrm{C} \mathrm{m}^{-2}$ ). See Table 57. [5, 25

$L_{d, 1 h}$ Fuel loading: Dead 1-hour fuels $\left(\mathrm{kg} \mathrm{C} \mathrm{m}^{-2}\right)$. See Table [57. + subscript, used by MC-Fire, indicates that fuel loading

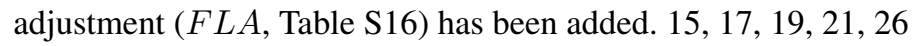

$L_{d, 10 h}$ Fuel loading: Dead 10-hour fuels $\left(\mathrm{kg} \mathrm{C} \mathrm{m}^{-2}\right)$. See Table 57. + subscript, used by MC-Fire, indicates that fuel loading adjustment (FTA, Table \$16) has been added. 15, [17, 211, 23]

$L_{d, 100 h}$ Fuel loading: Dead 100-hour fuels $\left(\mathrm{kg} \mathrm{C} \mathrm{m}^{-2}\right)$. See Table [S7. + subscript, used by MC-Fire, indicates that fuel loading

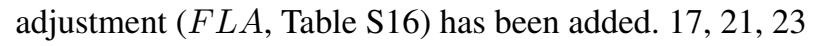

$25 L_{d, 1000 h}$ Fuel loading: Dead 1000-hour fuels $\left(\mathrm{kg} \mathrm{C} \mathrm{m}^{-2}\right)$. See Table $57 .+$ subscript, used by MC-Fire, indicates that fuel

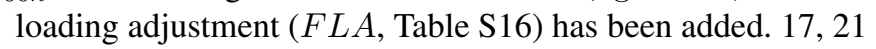

$L_{d c, n}$ Fuel loading: Combined dead fuels not including mineral content $\left(\mathrm{kg}\right.$ dry matter $\left.\mathrm{m}^{-2}\right)$. Note that the units of $L_{d c, n}$ are different from the other fuel loading variables, which use $\mathrm{kg} \mathrm{C} \mathrm{m}^{-2}$. See Table STI. [, 201, 25]

$L_{+d c, n, E}$ Fuel loading: Combined dead fuels not including mineral content, for use in energy release equations $\left(\mathrm{kg} \mathrm{C} \mathrm{m}^{-2}\right)$. Used by MC-Fire. See Table ST] [1]

$L_{+d c, n, R}$ Fuel loading: Combined dead fuels not including mineral content, for use in rate of spread equations $\left(\mathrm{kg} \mathrm{C} \mathrm{m}^{-2}\right)$. Used by MC-Fire. See Table ST]. 2]

$L_{d, g}$ Fuel loading: Dead grass $\left(\mathrm{kg} \mathrm{C} \mathrm{m}^{-2}\right)$. See Table $\mathbf{5 7}$. [15 
$L_{d+l, g}$ Fuel loading: Grass $\left(\mathrm{kg} \mathrm{C} \mathrm{m}^{-2}\right)$. See Table \$77. B0

$L_{d, \text { litter }}$ Fuel loading: Aboveground litter $\left(\mathrm{kg} \mathrm{C} \mathrm{m}^{-2}\right)$. ㄸ4, [15, 20]

$L_{d, S O M 1}$ Fuel loading: Soil organic matter in top (first) layer, i.e., organic horizon $\left(\mathrm{kg} \mathrm{C} \mathrm{m}^{-2}\right)$. See Table \$7]. प]

$L_{d u f f}$ Fuel loading: Duff $\left(\mathrm{kg} \mathrm{C} \mathrm{m}^{-2}\right)$. 144, 15]

$5 \quad L_{l}$ Fuel loading: Live biomass $\left(\mathrm{kg} \mathrm{C} \mathrm{m}^{-2}\right)$. See Table [57, [15, 25]

$L_{+l c, n}$ Fuel loading: Combined live fuels not including mineral content $\left(\mathrm{kg} \mathrm{C} \mathrm{m}^{-2}\right)$. Used by MC-Fire. See Table $\mathbf{S T l}$. 201

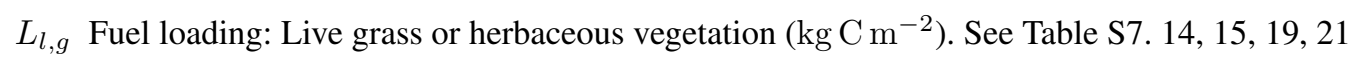

$L_{l, \text { leaf }}$ Fuel loading: Live leaves $\left(\mathrm{kg} \mathrm{C} \mathrm{m}^{-2}\right)$. See Table [57. [4, [5]

$L_{l, s}$ Fuel loading: Live shrubby vegetation $\left(\mathrm{kg} \mathrm{C} \mathrm{m}^{-2}\right)$. See Table S7. 201

$L_{l, \text { stem }}$ Fuel loading: Live stems $\left(\mathrm{kg} \mathrm{C} \mathrm{m}^{-2}\right)$. See Table [S7. [4, [15

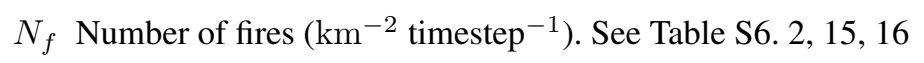

$N I$ Nesterov Index $\left({ }^{\circ} \mathrm{C}^{2}\right)$, a proxy for fuel moisture: $N I=\sum_{d} T_{\max , \mathrm{d}}\left(T_{\max , \mathrm{d}}-T_{\text {dew,d }}\right)$, where the summation occurs over

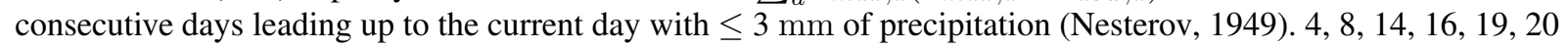

$\nabla \rrbracket_{\text {max }, y}$ Maximum Nesterov Index $\left(\mathbb{} \square,{ }^{\circ} \mathrm{C}^{2}\right)$ in the fire year. "Fire year" is defined for each grid cell so as to avoid splitting its fire season in two. See Knorr et al. (2014). 미

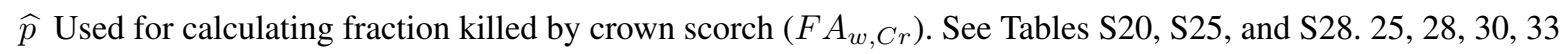

$P_{i}$ Probability of fire (unitless). See Table \$1]. 때, 표, ㅁ

$P_{i, h}$ Probability of fire ignited by humans (unitless). See Table $\mathbf{S 1}$. [1]

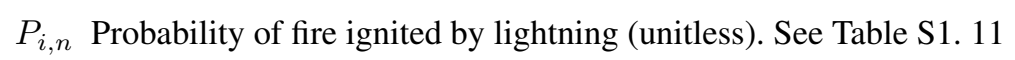

$20 P_{m}$ Probability of mortality from fire. Used by MC-Fire. See Table [S15. 25, [32]

$\widehat{p a r}_{1}$ Used for calculating tree diameter at breast height (DBH). See Tables $\mathbf{5 2 0}, \mathbf{5 2 5}$, and $\mathbf{5 2 8}$. [25, [28, [3]-[33]

$\widehat{\text { arr }_{2}}$ Used for calculating bark thickness (BT). See Tables $\$ 20$ and $\mathbf{5 2 8}$. [25, [28, [1], [3]

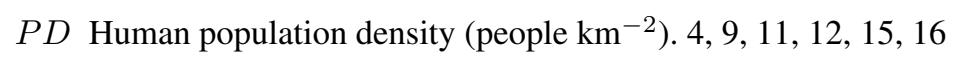

PET Potential evapotranspiration (mm). एप

$25 q$ Probability that a fire is extinguished on any given day (unitless). See Table [52. [1], ㅁ2, ㄷ, ㅁ

$Q_{i g}$ Heat of fuel pre-ignition $\left(\mathrm{kJ} \mathrm{kg}^{-1}\right)$. See Table $\mathbf{5 1 ]}$. [15, [2]

$\widehat{r}$ Resistance parameter for calculation of combustion and mortality. See Table [224. 22, 24, 30]

$\widehat{\sqrt{\mathrm{CH}}}$ Resistance parameter for calculation of crown scorch. See Tables [S20, S25, and [528, [25, [28, [30, [3]

$R_{d}$ Daily precipitation (mm). ㅁ, 26 


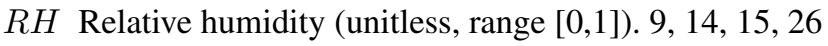

$R H_{30}$ Relative humidity, 30-day running mean (unitless, range [0,1]). ㄸ4

$R_{m}$ Monthly precipitation (mm). [9

ROS Rate of fire spread $\left(\mathrm{m} \mathrm{s}^{-1}\right)$. See Table [55]. [5, 201]

$5 R O S_{b}$ Rate of fire spread, backward (i.e., upwind; $\mathrm{m} \mathrm{s}^{-1}$ ). See Table [5. 메, [15]

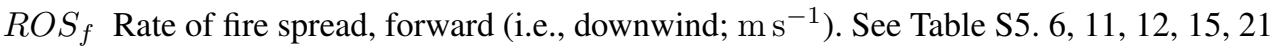

$\widehat{R O S}_{\text {max }}$ Maximum rate of forward (i.e., downwind) fire spread $\left(\mathrm{m} \mathrm{s}^{-1}\right)$. See Tables $\left.\$ 7\right]$ and $\mathbf{S T 8}$. 27, 28]

$R_{r}$ Precipitation rate $\left(\mathrm{mm} \mathrm{d}^{-1}\right)$. 144

$R_{t}$ Duration of precipitation (h). 26

$S$ General anthropogenic suppressive effect (unitless, value 0 or 1 ) on fire occurrence. Used by MC-Fire. See Table \$2. ए2, ए6

$s$ Slope angle (deg). [15, 201]

$S_{\theta}$ Suppressive effect of soil moisture (dimensionless, range [0,1]). Additional subscripts denote model-specific functions. See Table [54. [4, [5]

$S_{\text {bare }}$ Suppressive effect of bare ground cover (dimensionless, range [0,1]). See Table \$44. [4]

$S_{\text {env }}$ Suppressive effect (unitless, range [0,1]) of environmental conditions on number of fires, burned area per fire, total gridcell burned area, and/or fire intensity. See Table 54. [1, 때, ㅁ, 미

$S_{e n v, n f}$ Suppressive effect (unitless, range [0,1]) of environmental conditions on number of fires (or for CTEM, the fraction

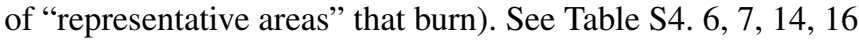

$S_{G D P, b a}$ Suppressive effect (unitless, range [0,1]) of gross domestic product per capita $\left(G D P_{p d}\right)$ on burned area per fire

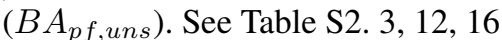

$S_{G D P, n f}$ Suppressive effect (unitless, range [0,1]) of gross domestic product per capita (GDP $\left.P_{p d}\right)$ on number of fires. See

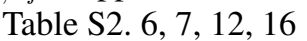

$S_{\square}$ Suppressive effect of fuel loading (dimensionless, range [0,1]). Additional subscripts denote model-specific functions. See Table 54. [44, [6, ㅁ]

$S_{P D, b a}$ Suppressive effect (unitless, range $\left.[0,1]\right)$ of population density $(\mathbb{P D})$ on burned area per fire $\left(B A_{p f, u n s}\right)$. See Table [S2, [1, [12, 16

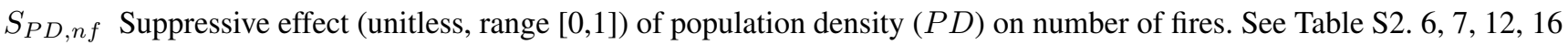

$S_{\mathbb{E R}]}$ Suppressive effect of relative humidity (dimensionless, range [0,1]). Additional subscripts denote model-specific functions. See Table [4. [4, [15]

$S_{T}$ Suppressive effect of temperature (dimensionless, range [0,1]). Additional subscripts denote model-specific functions. See Table [54. [44, [5]

$S_{G F E D}$ Scaling factor for number of fires, specific to each GFED region (van der Werf et al, 2006). Used by ORCHIDEESPITFIRE. See Table [54. [4, [35] 
SH Scorch height (m). See Table \$15. [4, [25]

$T_{\text {dew }, d}$ Daily dew-point air temperature $\left({ }^{\circ} \mathrm{C}\right) .8$, , ए9

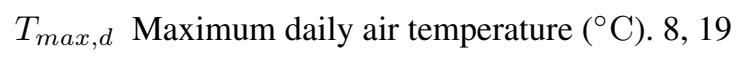

$T$ Air temperature $\left({ }^{\circ} \mathrm{C}\right)$. ㄸ4

$5 \quad t_{d l}$ Duration of daylight (h). 26

$t_{\text {fire }}$ Fire duration (s). See Table [55. [2, [15]

$t_{m}$ Month length (d). 미

$T F B B$ Total fuel bed biomass ( $\mathrm{T} \mathrm{DM} \mathrm{ac}^{-1}$ ). Alternative measure of total fuel loading, used only in calculation of depth by MC-Fire. See Table S16. 26

10 TSF Time since last fire (yr). 15

$T S R$ Time since last rainfall (d). 26

$W$ Wind speed at $10 \mathrm{~m}$ elevation $\left(\mathrm{m} \mathrm{s}^{-1}\right)$. [15, 21], 25, 26

$W^{\prime}$ Wind speed at elevation relevant for fire model $\left(\mathrm{ft} \mathrm{min}^{-1}\right)$. See Table $\mathbf{S W}$. 2]1

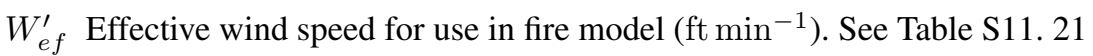

$15 W_{e f, m p h}^{\prime}$ Effective wind speed for use in fire model $\left(\mathrm{mi} \mathrm{h}^{-1}\right)$. See Table $\mathrm{SW}$. 21]

$w f$ Fraction of vegetated area ("foliar projective cover;" unitless) that is a woody PFT. $\mathbb{R}$

$\widehat{W R F}$ PFT-specific wind reduction factor (unitless) used by MC-Fire. See Table [27. [2], 132 


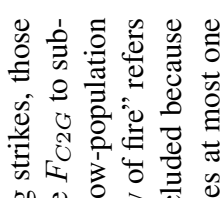
on 0

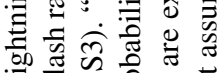

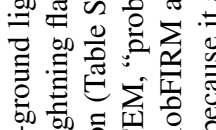

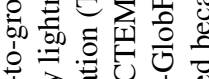

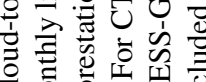

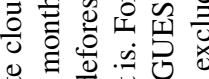
密

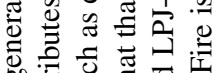

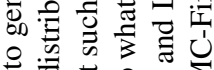

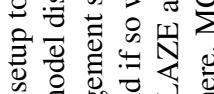

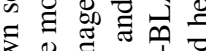

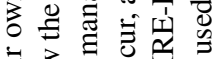

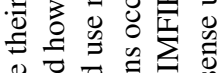

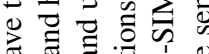

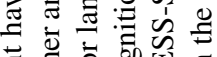

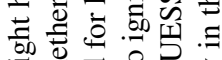

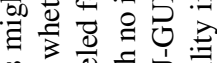

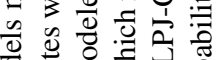

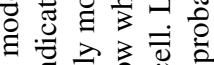

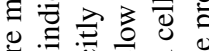
政

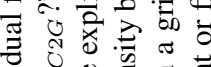

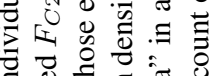

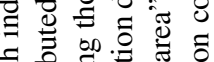

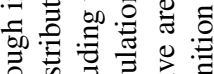

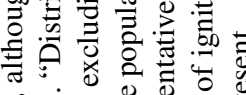

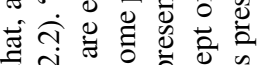

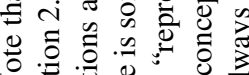
₹

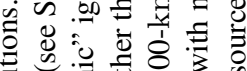

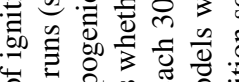

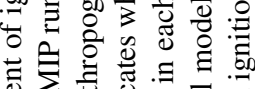

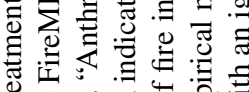

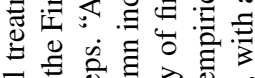

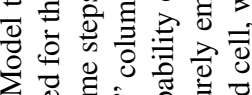

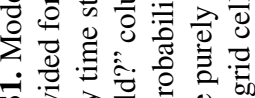

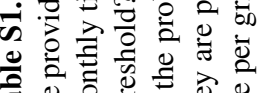

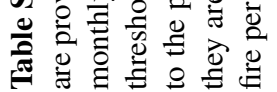

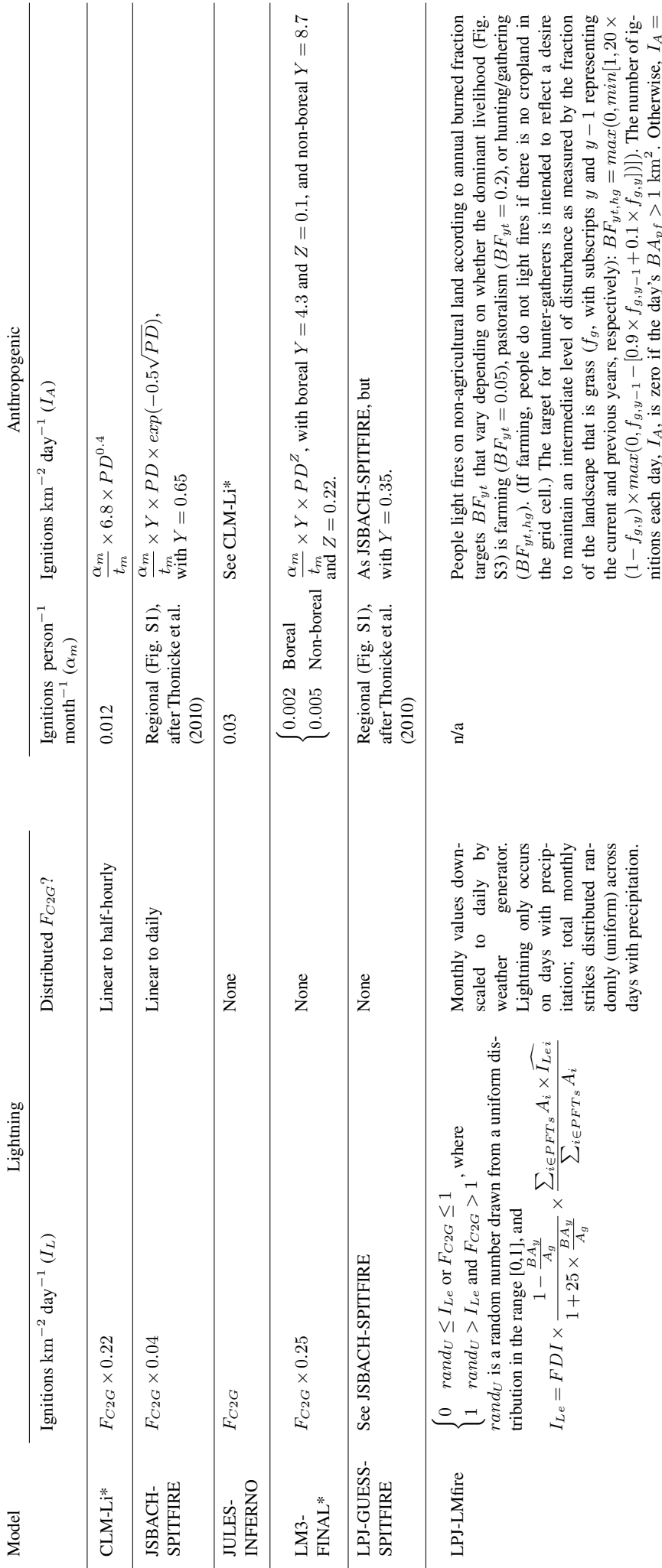

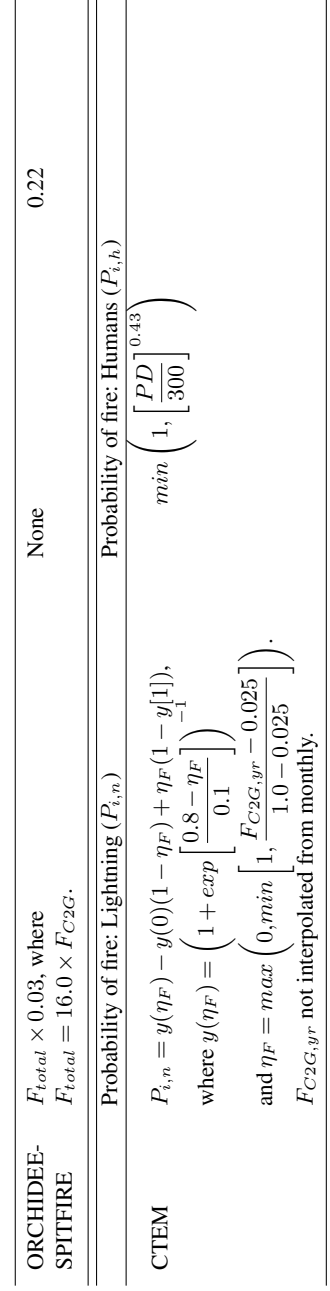




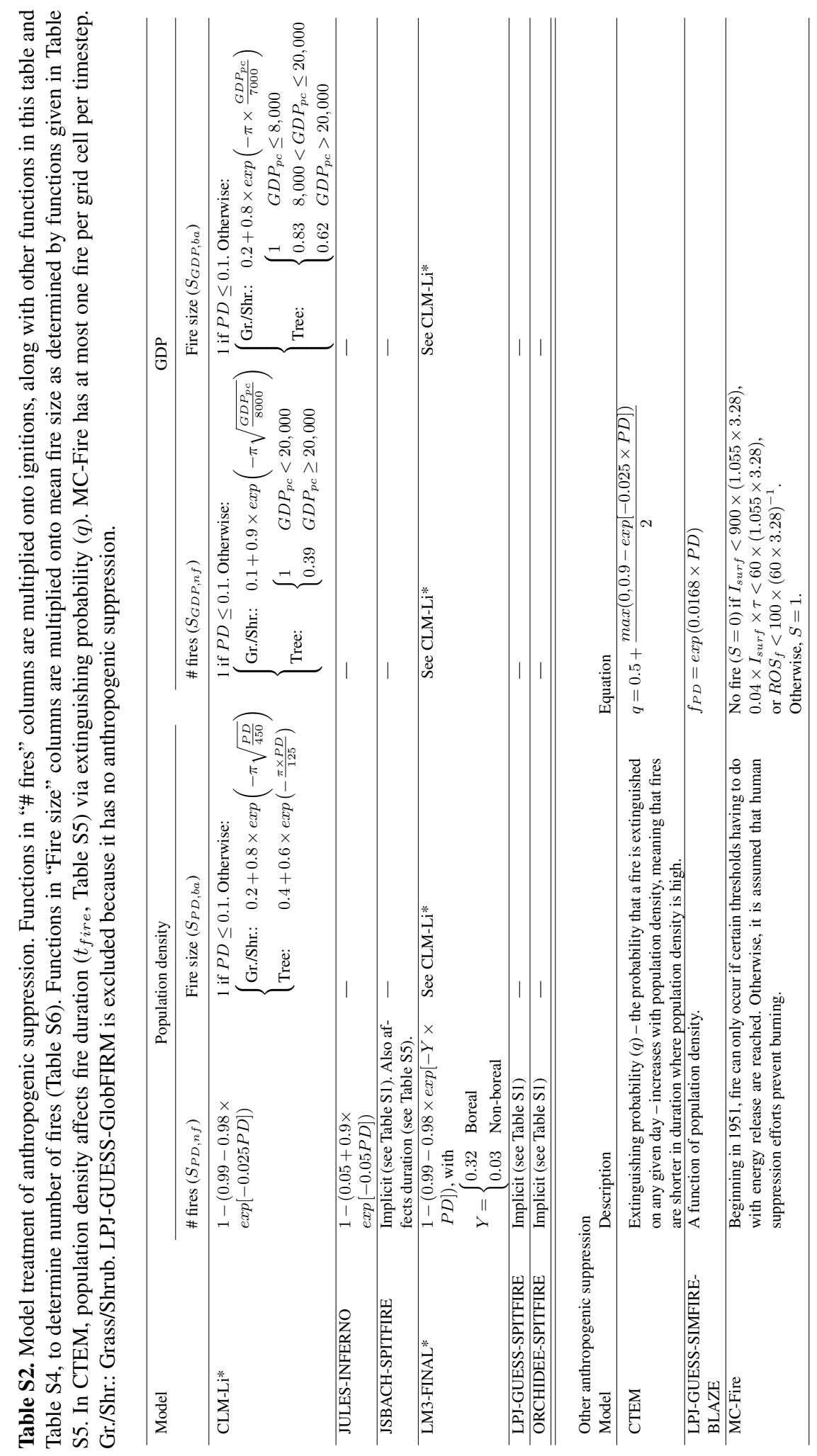




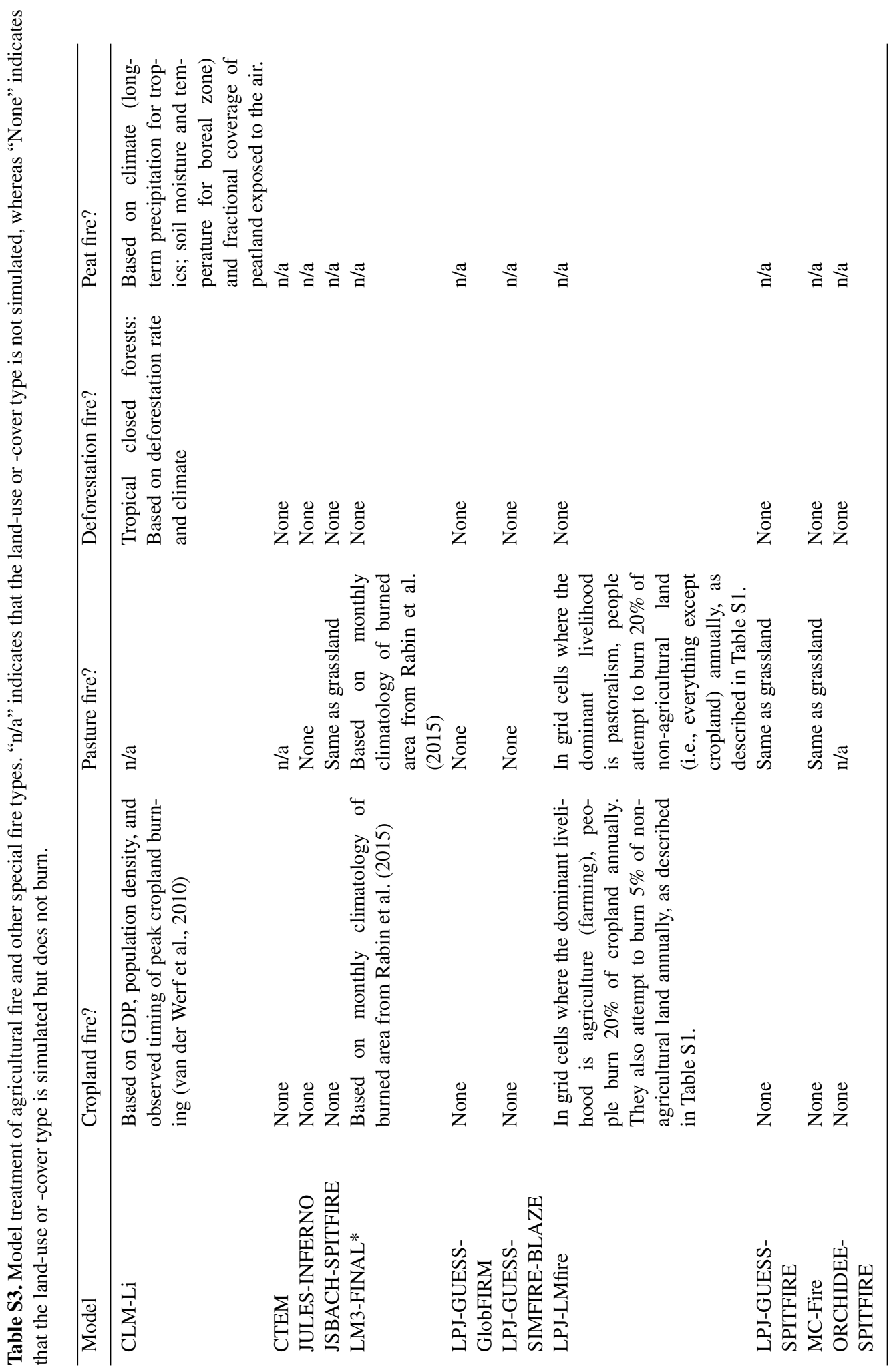


Table S4. How do environmental conditions and/or region of the world affect burning? $S_{e n v, n}$ affects the fraction of ignitions becoming

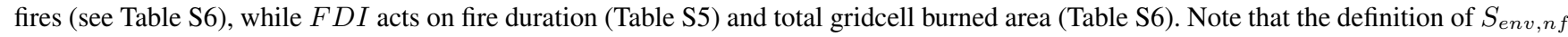
does not apply well to CTEM, where the maximum number of fires is essentially set at the number of "representative areas" that fit in a grid cell. Instead, consider $S_{\text {env,n }}$ a measure of the fraction of representative areas that burn.

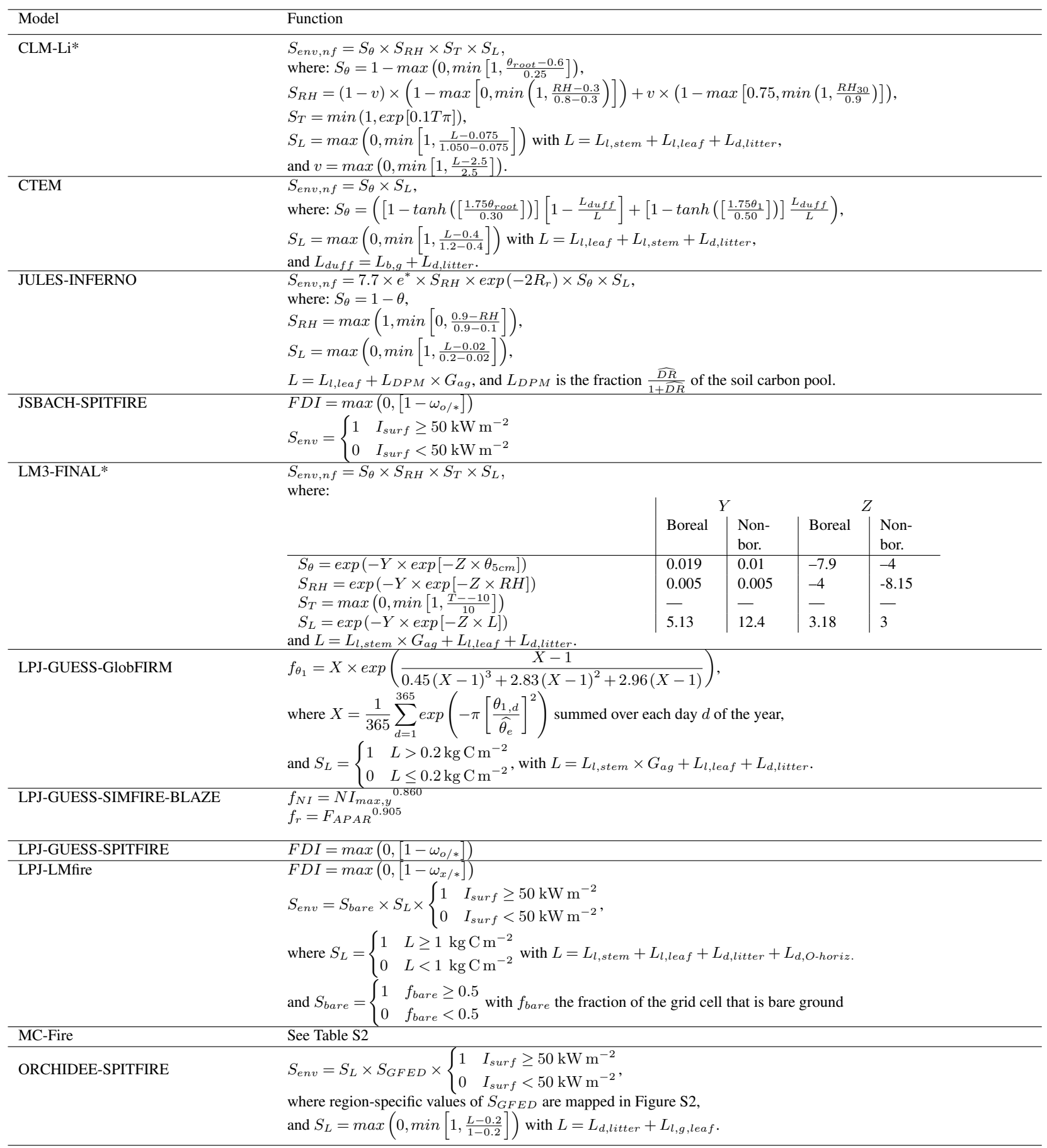




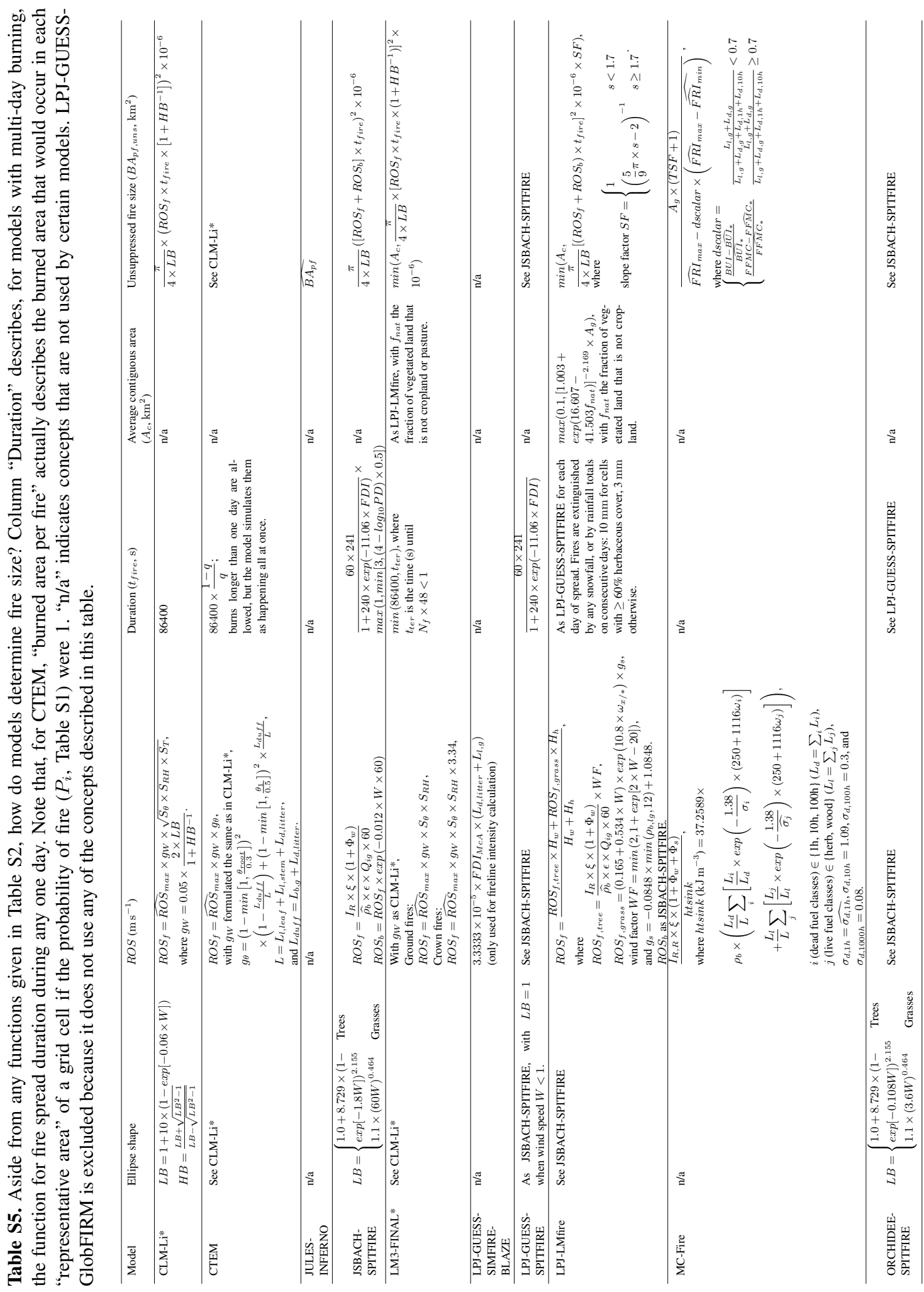


Table S6. How do the different models calculate total gridcell burned area $(\mathbb{B A})$ ? Units are $\mathrm{km}^{2}$ per timestep. Note that this does not include special fire types described in Table $\mathbf{5 3}$.

\begin{tabular}{|c|c|c|}
\hline Model & Timestep & Description \\
\hline CLM-Li* & Half-hourly & 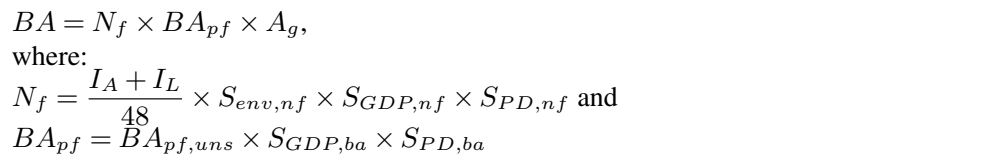 \\
\hline CTEM & Daily & 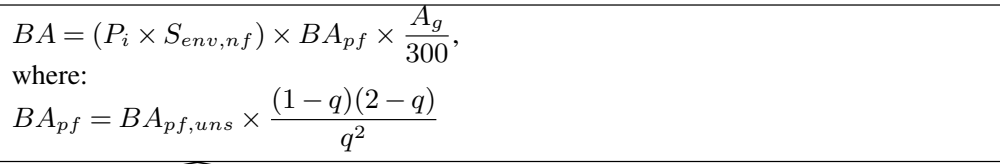 \\
\hline JULES-INFERNO & Half-hourly & 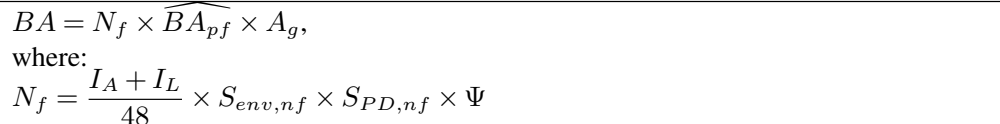 \\
\hline JSBACH-SPITFIRE & Daily & 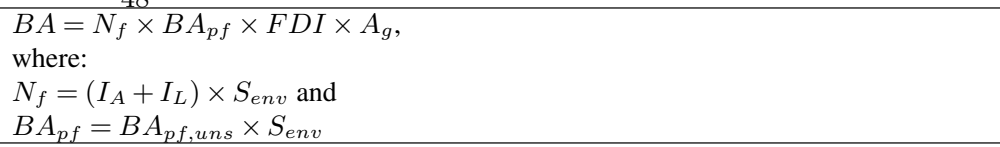 \\
\hline LM3-FINAL* & Half-hourly & See CLM-Li* \\
\hline LPJ-GUESS-GlobFIRM & Annual & $B A=\delta_{\theta_{1}} \times\left[S_{]} \times A_{g}\right.$ \\
\hline $\begin{array}{l}\text { LPJ-GUESS-SIMFIRE- } \\
\text { BLAZE }\end{array}$ & Monthly & 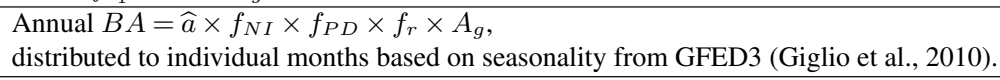 \\
\hline LPJ-GUESS-SPITFIRE & Daily & 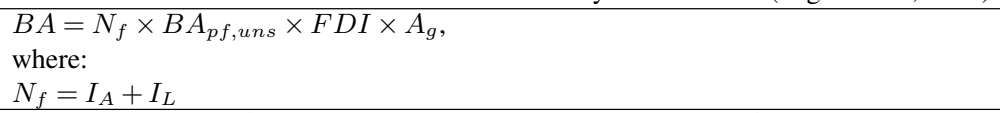 \\
\hline LPJ-LMfire & Daily & 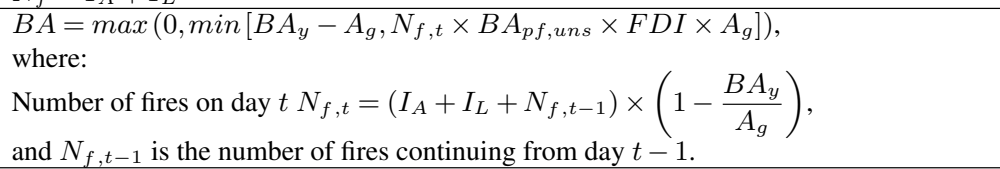 \\
\hline MC-Fire & Monthly & $\square \times B A_{p f, \text { uns }}$ \\
\hline ORCHIDEE-SPITFIRE & Daily & See JSBACH-SPITFIRE. \\
\hline
\end{tabular}


Table S7. How do the different models calculate fuel loads?

\begin{tabular}{|c|c|c|c|c|c|}
\hline Model & Description & $G_{a d}$ & & $G_{b d}$ & \\
\hline CLM-Li* & $\begin{array}{l}\text { CLM keeps track of live leaf, stem (all of which is aboveground), and root carbon pools, as well as } \\
\text { intermediate transfer and storage (T\&S) carbon pools. Dead carbon is comprised of leaf and woody litter } \\
\text { pools. All except live roots can be combusted when fire occurs (Table SI2), and all pools experience } \\
\text { fire-induced mortality, according to PFT- and tissue-specific fractions. An additional mortality factor } \\
\text { describes the transfer of biomass from sapwood ("live stem") to heartwood ("dead stem") with fire. }\end{array}$ & $\begin{array}{l}1 \\
0 \\
1 \\
1\end{array}$ & $\begin{array}{l}\text { Leaf } \\
\text { Root } \\
\text { Stem } \\
\text { T\&S }\end{array}$ & $\begin{array}{l}0 \\
1 \\
0 \\
1\end{array}$ & $\begin{array}{l}\text { Leaf } \\
\text { Root } \\
\text { Stem } \\
\text { T\&S }\end{array}$ \\
\hline CTEM & $\begin{array}{l}\text { CTEM tracks carbon in its three live vegetation components (leaves, stem, and roots) and two dead } \\
\text { carbon pools (litter and soil carbon). Specified fractions of leaves, aboveground stem, and litter pools } \\
\text { are combusted, and release emissions to the atmosphere, when fire occurs (Table } \mathbf{S 1 2} \text { ). In addition, fire } \\
\text { generates litter due to plant mortality based on specified fractions of leaves, stems, and roots (Table } \\
\text { S14). }\end{array}$ & $\begin{array}{l}1 \\
0 \\
1\end{array}$ & $\begin{array}{l}\text { Leaf } \\
\text { Root } \\
\text { Stem }\end{array}$ & $\begin{array}{l}0 \\
1 \\
1\end{array}$ & $\begin{array}{l}\text { Leaf } \\
\text { Root } \\
\text { Stem }\end{array}$ \\
\hline $\begin{array}{l}\text { JSBACH- } \\
\text { SPITFIRE }\end{array}$ & $\begin{array}{l}\text { Live biomass in JSBACH is divided into green, wood, and reserve pools. Dead biomass contributes to } \\
\text { the green and wood litter pools. Combustion affects aboveground biomass only, which consists of } 70 \% \\
\text { of the live woody pool and } 50 \% \text { of the live green pool (plus equivalent fractions of the litter derived } \\
\text { from those pools). Aboveground fuel is partitioned into fuel class sizes after Thonicke et all (DOIII): } \\
\text { 1-hour fuel (leaves and twigs) is all green biomass plus } 4.5 \% \text { of woody biomass, } 10 \text {-hour fuel (small } \\
\text { branches) is } 7.5 \% \text { of woody biomass, } 100 \text {-hour fuel (large branches) is } 21 \% \text { of woody biomass, and } \\
1000 \text {-hour fuel (trunks) is } 67 \% \text { of woody biomass. }\end{array}$ & $\begin{array}{l}0.5 \\
0.7\end{array}$ & $\begin{array}{l}\text { Green } \\
\text { Woody }\end{array}$ & $\begin{array}{l}0.5 \\
0.3\end{array}$ & $\begin{array}{l}\text { Green } \\
\text { Woody }\end{array}$ \\
\hline $\begin{array}{l}\text { JULES- } \\
\text { INFERNO }\end{array}$ & 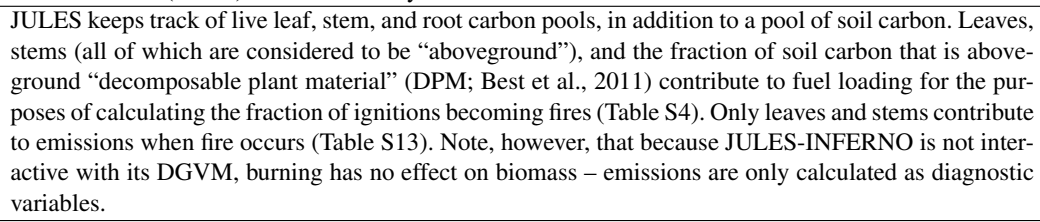 & $\begin{array}{l}1 \\
1 \\
0 \\
0.7\end{array}$ & $\begin{array}{l}\text { Leaf } \\
\text { Stem } \\
\text { Root } \\
\text { DPM }\end{array}$ & $\begin{array}{l}0 \\
0 \\
1 \\
0.3\end{array}$ & $\begin{array}{l}\text { Leaf } \\
\text { Stem } \\
\text { Root } \\
\text { DPM }\end{array}$ \\
\hline LM3-FINAL* & $\begin{array}{l}\text { LM3 tracks five live vegetation carbon pools: leaves, hardwood, sapwood, labile carbon, and fine roots. } \\
\text { Here, "stem" biomass refers to the hardwood, sapwood, and labile carbon pools. LM3 also includes leaf } \\
\text { and coarse wood litter pools. } 80 \% \text { of stem biomass is aboveground and therefore considered part of fuel } \\
\text { loading for the purposes of } S_{I} \text {, along with live leaves and leaf litter. (Although only aboveground stem } \\
\text { biomass matters for fuel loading, all stem biomass is susceptible to combustion and mortality.) }\end{array}$ & $\begin{array}{l}0 \\
1 \\
0.8\end{array}$ & $\begin{array}{l}\text { Fine root } \\
\text { Leaf } \\
\text { Stem }\end{array}$ & $\begin{array}{l}1 \\
0 \\
0.2\end{array}$ & $\begin{array}{l}\text { Fine root } \\
\text { Leaf } \\
\text { Stem }\end{array}$ \\
\hline $\begin{array}{l}\text { LPJ-GUESS- } \\
\text { GlobFIRM }\end{array}$ & $\begin{array}{l}\text { LPJ-GUESS partitions both live vegetation and litter C into branches, bark, trunks, leaves, and fine } \\
\text { roots. All except fine roots are combusted when fire occurs (Table } \mathbf{S T 2}) \text {, with fine roots getting killed } \\
\text { instead (Table } \mathbf{S T 4} \text { ). In these tables, "aboveground stem" refers to biomass in branches, bark, and trunks. }\end{array}$ & $\begin{array}{l}0 \\
1 \\
1\end{array}$ & $\begin{array}{l}\text { Fine root } \\
\text { Leaf } \\
\text { Stem }\end{array}$ & $\begin{array}{l}1 \\
0 \\
0\end{array}$ & $\begin{array}{l}\text { Fine root } \\
\text { Leaf } \\
\text { Stem }\end{array}$ \\
\hline $\begin{array}{l}\text { LPJ-GUESS- } \\
\text { SIMFIRE- } \\
\text { BLAZE }\end{array}$ & $\begin{array}{l}\text { LPJ-GUESS partitions live vegetation carbon into heartwood, sapwood, leaves, and roots. Together, } \\
\text { heartwood and sapwood are referred to as stem biomass, all of which is considered to be above ground. } \\
\text { Stem biomass is further subdivided: } 5 \% \text { as branches, } 1 \% \text { as bark, and the remaining } 94 \% \text { as trunks. } \\
\text { Dead biomass is partitioned into metabolic C, structural C, and fine and coarse woody debris - together } \\
\text { referred to here as litter. All living and dead biomass, except belowground stem (i.e., coarse roots) and } \\
\text { fine roots, can be combusted when fire occurs (Table } \mathbf{S 1 2} \text { ). }\end{array}$ & $\begin{array}{l}0 \\
1 \\
1\end{array}$ & $\begin{array}{l}\text { Fine root } \\
\text { Leaf } \\
\text { Stem }\end{array}$ & $\begin{array}{l}1 \\
0 \\
0\end{array}$ & $\begin{array}{l}\text { Fine root } \\
\text { Leaf } \\
\text { Stem }\end{array}$ \\
\hline $\begin{array}{l}\text { LPJ-GUESS- } \\
\text { SPITFIRE }\end{array}$ & $\begin{array}{l}\text { LPJ-GUESS partitions live vegetation carbon into heartwood, sapwood, leaves, and roots. Together, } \\
\text { heartwood and sapwood are referred to as stem biomass - all of which is considered to be above ground } \\
\text { for fire modeling purposes. Leaf and woody litter pools are converted to fuel class loadings after Thon- } \\
\text { Icke et al. (20I0), as described for JSBACH-SPITFIRE. }\end{array}$ & $\begin{array}{l}0 \\
1 \\
1\end{array}$ & $\begin{array}{l}\text { Fine root } \\
\text { Leaf } \\
\text { Stem }\end{array}$ & $\begin{array}{l}1 \\
0 \\
0\end{array}$ & $\begin{array}{l}\text { Fine root } \\
\text { Leaf } \\
\text { Stem }\end{array}$ \\
\hline LPJ-LMfire & $\begin{array}{l}\text { Biomass pools and fuel loading are as described for LPJ-GUESS-SPITFIRE, with the addition of a } \\
\text { soil organic horizon (O-horiz.), which for fire modeling purposes is only considered when considering } \\
\text { whether there is enough fuel for fire to occur. The soil organic horizon is not combusted and does } \\
\text { not contribute to fuel moisture. However, it does serve to effectively remove fuel from other dead fuel } \\
\text { classes. }\end{array}$ & $\begin{array}{l}0 \\
1 \\
1\end{array}$ & $\begin{array}{l}\text { Fine root } \\
\text { Leaf } \\
\text { Stem }\end{array}$ & $\begin{array}{l}1 \\
0 \\
0\end{array}$ & $\begin{array}{l}\text { Fine root } \\
\text { Leaf } \\
\text { Stem }\end{array}$ \\
\hline MC-Fire & $\begin{array}{l}\text { Live fuel in MC-Fire consists of all live grass and tree biomass. Stem wood is partitioned into above- } \\
\text { ground and belowground biomass - and aboveground wood is partitioned into fine branches, large } \\
\text { branches, and trunks - based on equations in the vegetation model. Total dead fuel load is partitioned } \\
\text { into leaf litter (structural and metabolic carbon), standing dead grass, fine dead wood, and coarse dead } \\
\text { wood. The amounts of dead 1-hour }\left(L_{d, 1 h}\right), 10 \text {-hour }\left(L_{d, 10 h}\right), 100 \text {-hour }\left(L_{d, 100 h}\right) \text {, and } 1000 \text {-hour } \\
\left(L_{d, 1000 h}\right) \text { fuels are calculated by multiplying total dead fuel load by a respective dead fuel fraction: } \\
\left.D F F_{1 h}, D F F_{10 h}, \overline{D F F_{100 h}} \text {, and } \overline{D F F_{1000 h}} \text { (Table } \overline{D 27}\right) \text {. }\end{array}$ & & $\begin{array}{l}\text { Fine root } \\
\text { Leaf } \\
\text { Stem }\end{array}$ & $\begin{array}{l}1 \\
0 \\
G_{b g}, s\end{array}$ & $\begin{array}{l}\text { Fine root } \\
\text { Leaf } \\
\text { Stem }\end{array}$ \\
\hline $\begin{array}{l}\text { ORCHIDEE- } \\
\text { SPITFIRE }\end{array}$ & $\begin{array}{l}\text { Live biomass in ORCHIDEE is divided into leaves, sapwood, heartwood, fine roots, a reproductive } \\
\text { pool, and a carbohydrate reserve pool. The fraction of live biomass in all except heartwood is deter- } \\
\text { mined dynamically by an allocation scheme; heartwood is derived from sapwood, and together these } \\
\text { are referred to as "wood." Dead fuels are comprised of the fast and slow litter pools, named for their } \\
\text { respective rates of decomposition. The amount of each litter pool that is derived from each of the plant } \\
\text { biomass pools is calculated as a diagnostic based on conversion ratios. The loading of each fuel class } \\
\text { is calculated after Thonicke et al. (2010), similarly to JSBACH-SPITFIRE: Wood and carbohydrate re- } \\
\text { serves are divided into 1-hour fuel (4.5\%, plus all leaf and reproductive biomass), 10-hour fuel (7.5\%), } \\
\text { 100-hour fuel (21\%), and 1000-hour fuel (67\%). }\end{array}$ & & $\begin{array}{ll}\text { Fine root } \\
\text { Leaf } \\
\text { Stem }\end{array}$ & $\begin{array}{l}1 \\
0 \\
G_{b g}, s\end{array}$ & $\begin{array}{l}\text { Fine root } \\
\text { Leaf } \\
\text { Stem }\end{array}$ \\
\hline
\end{tabular}


Table S8. Constants relating to Rothermel-style processes. CLM-Li*, CTEM, LM3-FINAL*, JULES-INFERNO, and LPJ-GUESSGlobFIRM are excluded because they do not include these processes. 因: Fuel heat content $\left(\mathrm{kJ} \mathrm{kg}^{-1}\right)$. TS is rounded to five decimal places. Note that MC-FIRE uses $32 \mathrm{lb} . \mathrm{ft}^{-3}$ for $p_{p}$, which rounds to $513 \mathrm{~kg} \mathrm{~m}^{-3}$. "n/a" indicates variables that are not used by LPJ-GUESSSIMFIRE-BLAZE.

\begin{tabular}{llll}
\hline Model & G & pv & पTS \\
\hline JSBACH-SPITFIRE & 18,000 & 513 & 0.41739 \\
LPJ-GUESS-SIMFIRE-BLAZE & 20,000 & $\mathrm{n} / \mathrm{a}$ & $\mathrm{n} / \mathrm{a}$ \\
LPJ-GUESS-SPITFIRE & 18,000 & 513 & 0.41739 \\
LPJ-LMfire & 18,000 & 513 & 0.41739 \\
MC-FIRE & $\hat{6}$ & 513 & 0.41739 \\
ORCHIDEE-SPITFIRE & 18,000 & 513 & 0.41740 \\
\hline
\end{tabular}




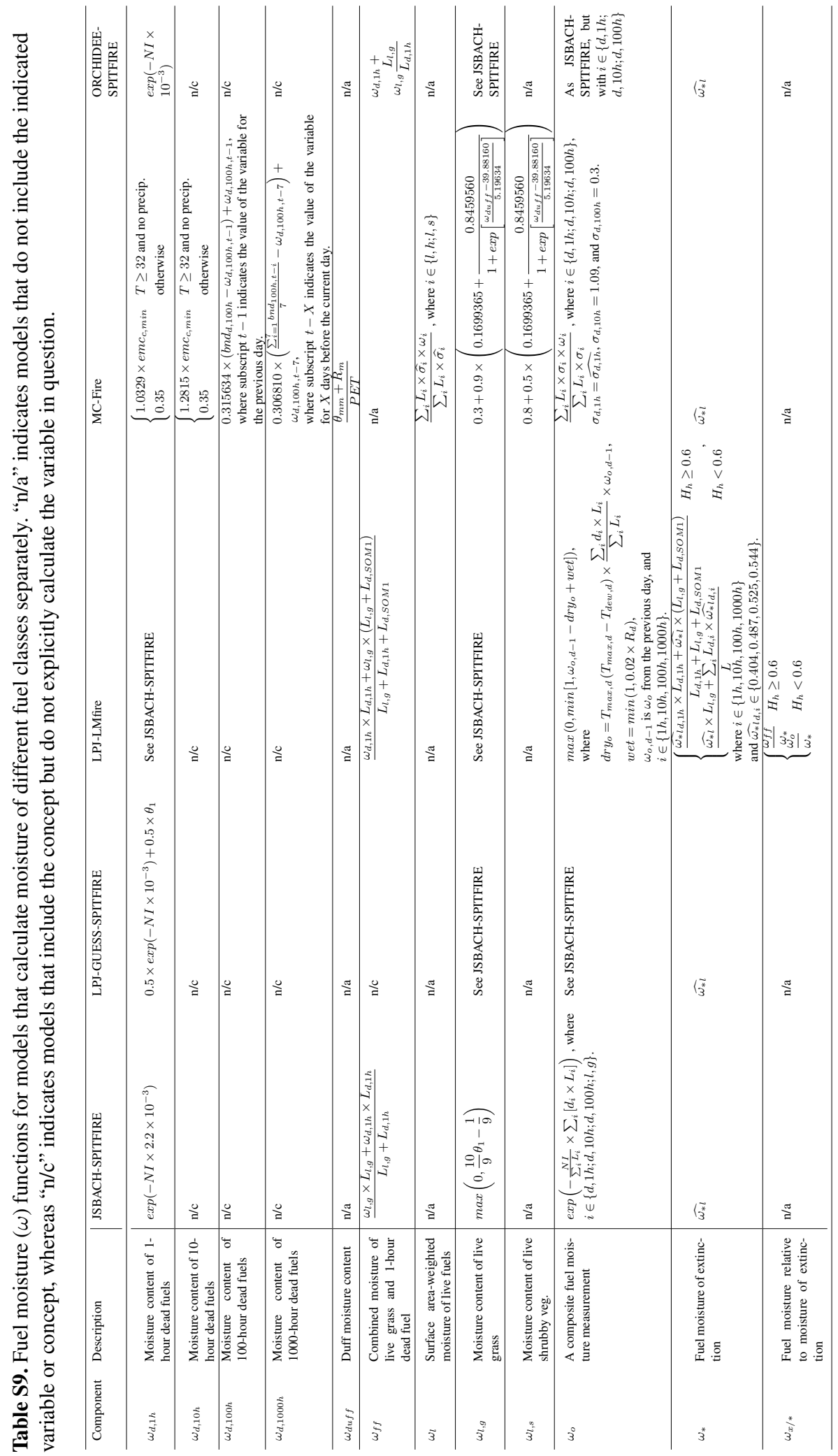


Table S10. Drying parameters for models that use them in calculating fuel moisture (Table 59). "n/a" indicates models that do not include the indicated variable.

\begin{tabular}{|c|c|c|c|c|}
\hline Variable & $\begin{array}{l}\text { JSBACH- } \\
\text { SPITFIRE }\end{array}$ & $\begin{array}{l}\text { LPJ-GUESS- } \\
\text { SPITFIRE }\end{array}$ & LPJ-LMfire & $\begin{array}{l}\text { ORCHIDEE- } \\
\text { SPITFIRE }\end{array}$ \\
\hline$d_{1 h}$ & $2.2 \times 10^{-3}$ & $1 \times 10^{-3}$ & $1.5 \times 10^{-3}$ & $\begin{array}{l}\text { See JSBACH- } \\
\text { SPITFIRE }\end{array}$ \\
\hline$d_{10 h}$ & $\sim 1.193 \times 10^{-4}$ & $5.42 \times 10^{-5}$ & $8.13 \times 10^{-5}$ & $\begin{array}{l}\text { See JSBACH- } \\
\text { SPITFIRE }\end{array}$ \\
\hline$d_{100 \mathrm{~h}}$ & $\sim 3.27 \times 10^{-5}$ & $1.49 \times 10^{-5}$ & $2.22 \times 10^{-5}$ & $\begin{array}{l}\text { See JSBACH- } \\
\text { SPITFIRE }\end{array}$ \\
\hline$d_{1000 h}$ & $\mathrm{n} / \mathrm{a}$ & & $1.5 \times 10^{-6}$ & $\mathrm{n} / \mathrm{a}$ \\
\hline$d_{l, g}$ & $\sim 2.2 \times 10^{-3}$ & $-\frac{\log \left(\omega_{l, g}\right)}{\Delta \nabla}$ & $\mathrm{n} / \mathrm{a}$ & $\begin{array}{l}\text { See JSBACH- } \\
\text { SPITFIRE }\end{array}$ \\
\hline
\end{tabular}




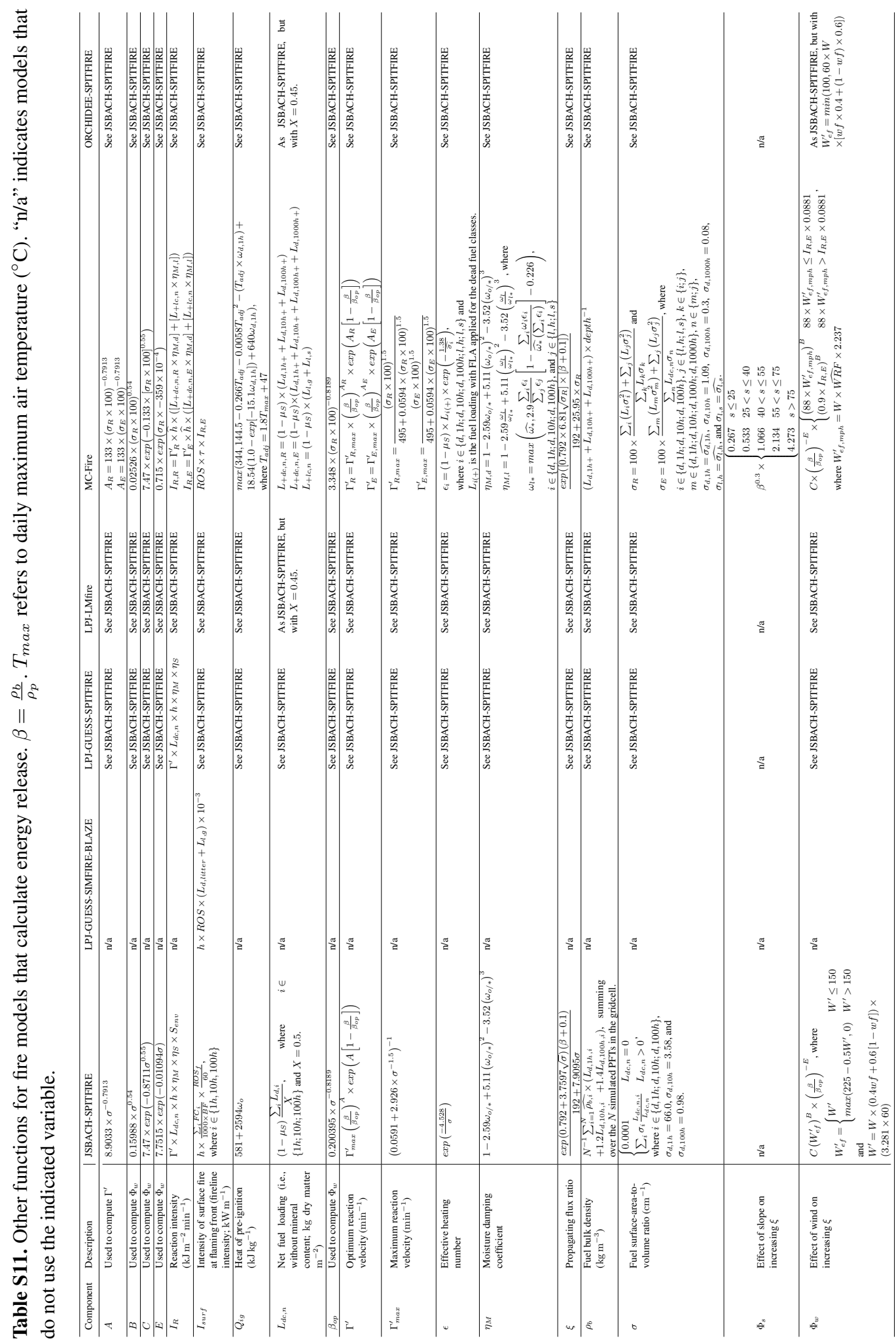




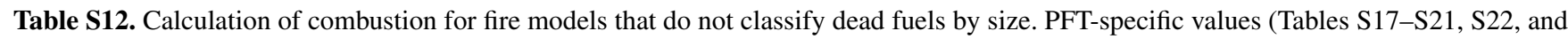
S24) are denoted with a "hat." "n/a" indicates models that do not include the indicated variable or concept. Models have n/a for FC $C_{l, g r a s s}$ if grass PFTs are not affected differently from woody PFTs.

\begin{tabular}{|c|c|c|c|c|c|c|}
\hline Component & CLM-Li* & CTEM & JULES-INFERNO & $\begin{array}{l}\text { LM3- } \\
\text { FINAL }\end{array}$ & $\begin{array}{l}\text { LPJ-GUESS- } \\
\text { GlobFIRM }\end{array}$ & LPJ-GUESS-SIMFIRE-BLAZE \\
\hline $\mathrm{FC}_{l, l e a}$ & $\widehat{F C_{l, l e a}}$ & $\widehat{F C_{l, \text { lea }}}$ & $\begin{array}{l}F C_{\text {l,leaf,min }}+(1-\theta) \\
\times\left(F C_{\text {l.leaf.max }}-F C_{\text {l.leaf.min }}\right)\end{array}$ & $\widehat{F C_{l, l e a}}$ & $1-$ 田 & $F A_{w, C a+C r} \times \begin{cases}0.02 & I_{\text {sur }} \leq 0.75 \\
0.05 & 0.75<I_{\text {sur }} \leq 3 \\
0.10 & 3<I_{\text {sur }} \leq 7 \\
0.60 & I_{\text {sur }}>7\end{cases}$ \\
\hline$F C_{l, \text { root }}$ & 0 & 0 & 0 & 0 & 0 & $F A_{w, C a+C r} \times \begin{cases}0 & I_{\text {sur }} \leq 0.75 \\
0.02 & 0.75<I_{\text {sur }} \leq 7 \\
0.04 & I_{\text {sur }}>7\end{cases}$ \\
\hline $\mathrm{FC}_{l, \text { stem }}$ & $\widehat{F C_{l, s t e m}}$ & $\begin{array}{l}\widehat{F C l, s t e m} \\
\times \widehat{G a d}\end{array}$ & $\begin{array}{l}F \bar{C}_{l, \text { stem,min }}+(1-\boldsymbol{\theta}) \\
\times\left(F \bar{C}_{l, \text { stem,man }}-F \bar{C}_{l, \text { stem.min }}\right)\end{array}$ & $\widehat{F C l, s t e m}$ & 1-回 & 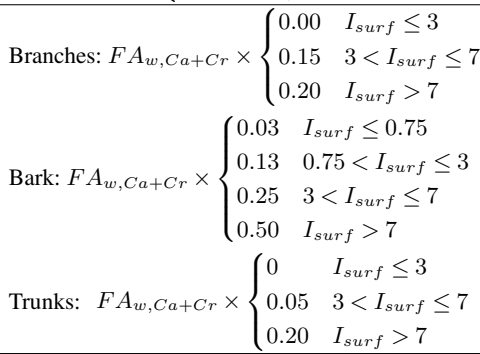 \\
\hline$F C_{l, g r a s s}$ & $\mathrm{n} / \mathrm{a}$ & $F C_{l, \text { leaf }}$ & $\mathrm{n} / \mathrm{a}$ & $\mathrm{n} / \mathrm{a}$ & $1-$ 回 & $0.99 \times G_{a d}$ \\
\hline$F C_{d, l e a}$ & 0.5 & $\widehat{F C_{d, l i t t e r}}$ & $\mathrm{n} / \mathrm{a}$ & $\widehat{F C \widehat{C_{d, l i t t e n}}}$ & $1-$ 田 & $\begin{cases}0.60 & I_{\text {sur }} \leq 0.75 \\
0.65 & 0.75<I_{\text {sur }} \leq 3 \\
0.85 & 3<I_{\text {sur }} \leq 7 \\
1.00 & I_{\text {sur }}>7\end{cases}$ \\
\hline$F C_{d, \text { stem }}$ & 0.35 & $F C_{d, l i t t e r}$ & $\mathrm{n} / \mathrm{a}$ & $F C_{d, \text { litten }}$ & $1-$ 回 & $\begin{array}{l}\text { Bark: See } F C_{\text {d,lea }} \\
\text { Branches, } \\
\text { trunks } \begin{cases}0.50 & I_{\text {surf }} \leq 0.75 \\
0.75 & 0.75<I_{\text {sur }} \leq 7 \\
0.80 & I_{\text {surf }}>7\end{cases} \end{array}$ \\
\hline$F C_{\text {d.root }}$ & $\mathrm{n} / \mathrm{a}$ & $\mathrm{n} / \mathrm{a}$ & $\mathrm{n} / \mathrm{a}$ & 0 & 0 & 0 \\
\hline
\end{tabular}




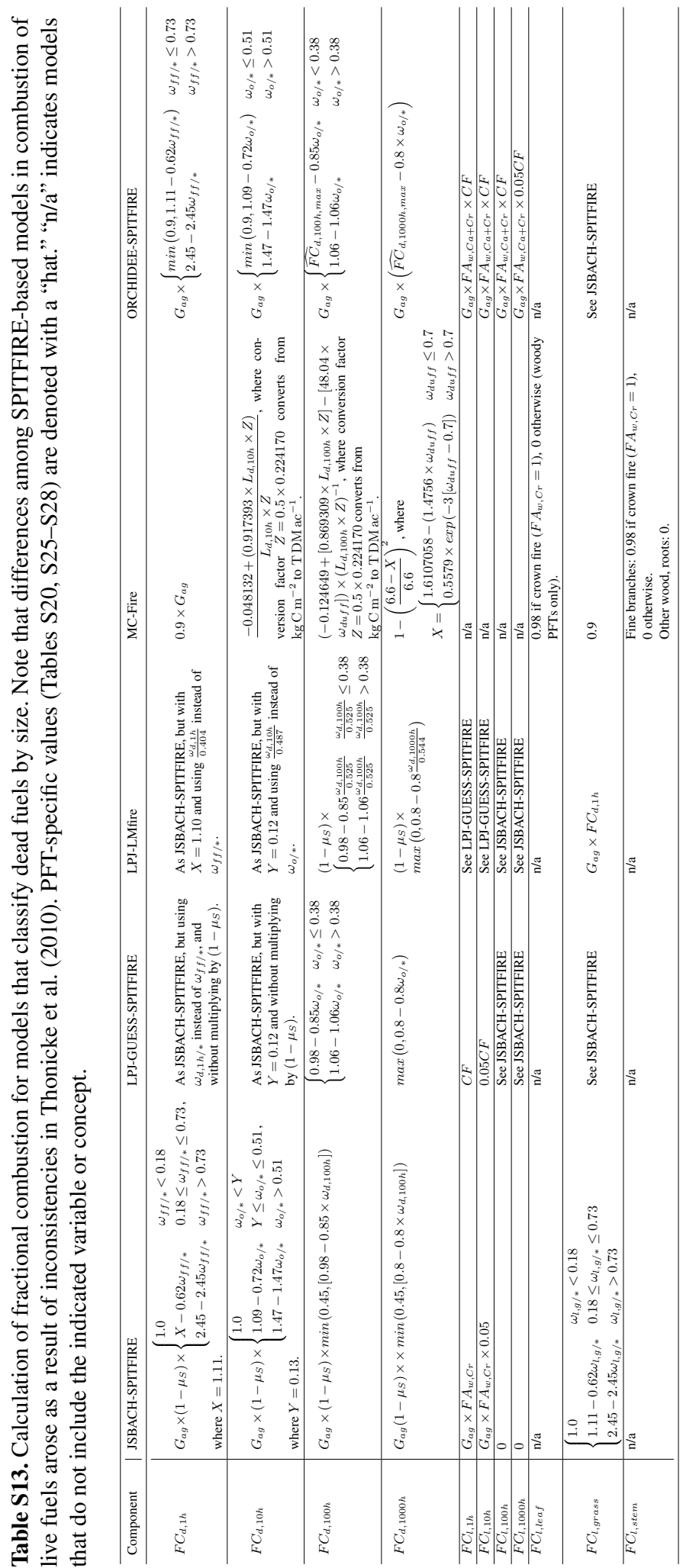


Table S14. Calculation of fire-induced mortality for models that do not use tree size and/or fire intensity as a driver. Note that this does not include biomass killed during combustion; for these purposes "mortality" refers to biomass transferred from living to dead or soil pools. JULES-INFERNO does not calculate fire-induced mortality and is thus excluded. PFT-specific values (Tables S17-S19) are denoted with a "hat." "n/a" indicates models that do not include the indicated variable or concept.

\begin{tabular}{|c|c|c|c|c|c|}
\hline Component & Description & CLM-Li* & CTEM & $\begin{array}{l}\text { LPJ-GUESS- } \\
\text { GlobFIRM }\end{array}$ & LM3-FINAL* \\
\hline FK lea & $\begin{array}{l}\text { Fractional mortality: } \\
\text { Leaves }\end{array}$ & $\left(1-\widehat{F C_{\text {l,lea }}}\right) \times \widehat{F K_{\text {lea }}}$ & $\widehat{F K_{\text {lea }}}$ & 0 & See CLM-Li* \\
\hline$F K_{\text {root }}$ & $\begin{array}{l}\text { Fractional mortality: } \\
\text { Roots }\end{array}$ & FKKroot & See CLM-Li* & 1-田 & See CLM-Li* \\
\hline FK $K_{\text {stem }}$ & $\begin{array}{l}\text { Fractional mortality: } \\
\text { Stem }\end{array}$ & 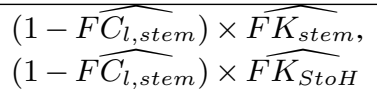 & FK, stem & 0 & See CLM-Li* \\
\hline
\end{tabular}




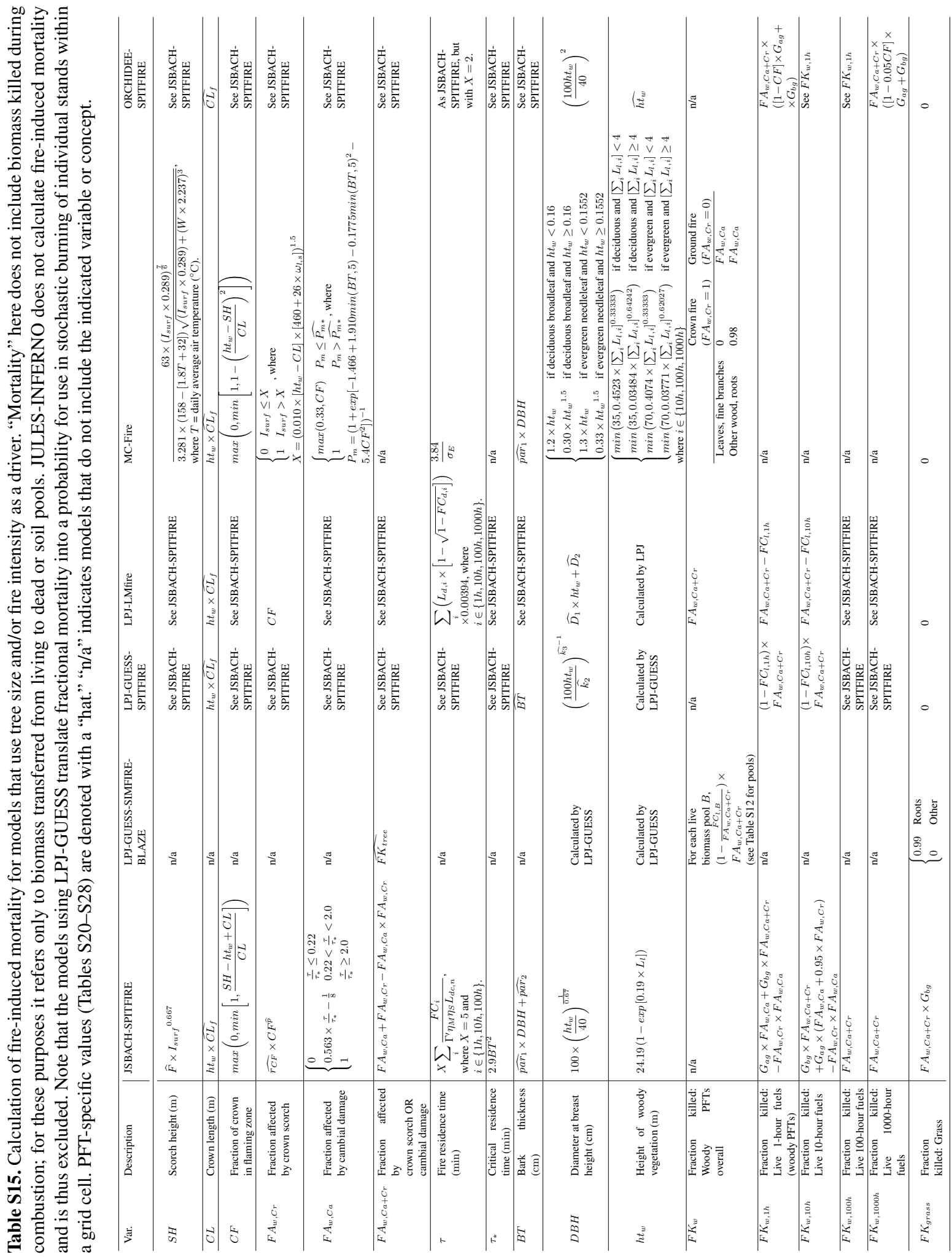




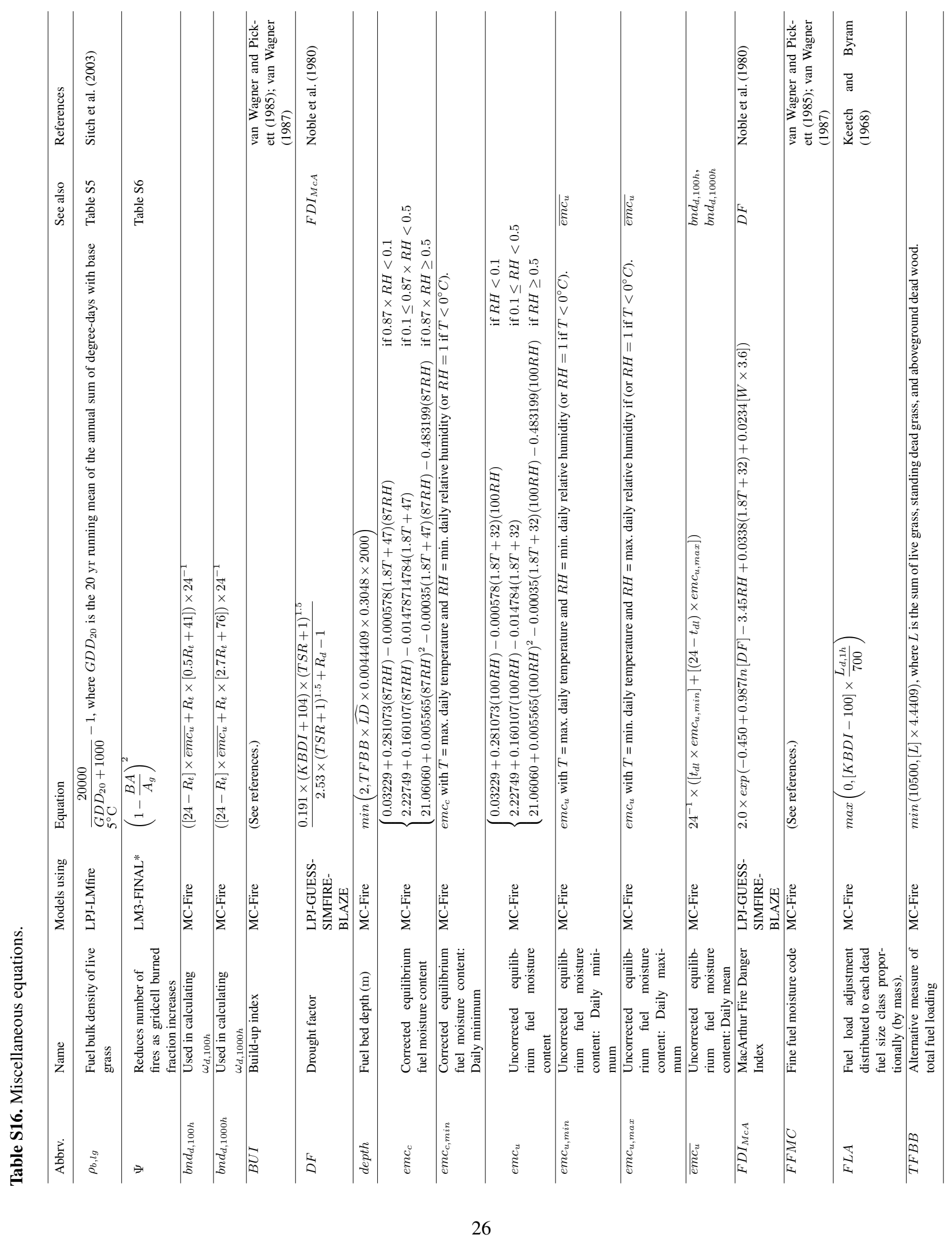


Table S17. PFT-specific information for CLM-Li*: PFT-specific fractional combustion completeness factors for leaves (FC $\widehat{F l, l e a t})$, stems $\left(\widehat{F C_{l, s t e m}}\right)$, and transfer and storage carbon $\left(\widehat{F C_{l, t s}}\right)$; mortality factors for leaves $\left(\widehat{F K_{\text {lea }}}\right)$, stems $\left(\widehat{F K_{\text {stem }}}\right)$, roots $\left(\widehat{F K_{\text {raot }}}\right)$, and transfer and storage carbon $\left(\widehat{F K_{t s}}\right)$. An additional "mortality" factor $\widehat{F K_{S t o H}}$ describes the fire-induced transfer of carbon from sapwood to heartwood (i.e., from the live portion of the stem to the dead portion) after previous combustion and mortality factors are applied. $\underline{R} O S_{m a x}$ : Maximum rate of spread $\left(\mathrm{m} \mathrm{s}^{-1}\right)$. Abbreviations in PFT names: $\mathrm{N}$ (needleleaf), B (broadleaf), E (evergreen), D (deciduous), T (tree), S (shrub).

\begin{tabular}{|c|c|c|c|c|c|c|c|c|c|}
\hline PFT & $\widehat{F C_{l, l e a t}}$ & FC & $\widehat{F C_{l, t s}}$ & $\widehat{F K_{\text {lea }}}$ & $\widehat{F K_{\text {stem }}}$ & $\widehat{F K_{\text {root }}}$ & $\widehat{F K_{t s}}$ & 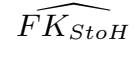 & $\widehat{R O S_{\max }}$ \\
\hline NET Temperate & 0.8 & 0.27 & 0.45 & 0.8 & 0.13 & 0.13 & 0.5 & 0.37 & 0.3 \\
\hline NET Boreal & 0.8 & 0.3 & 0.5 & 0.8 & 0.15 & 0.15 & 0.55 & 0.4 & 0.32 \\
\hline NDT Boreal & 0.8 & 0.3 & 0.5 & 0.8 & 0.15 & 0.15 & 0.55 & 0.4 & 0.32 \\
\hline BET Tropical & 0.8 & 0.27 & 0.45 & 0.8 & 0.13 & 0.13 & 0.5 & 0.37 & 0.3 \\
\hline BET Temperate & 0.8 & 0.27 & 0.45 & 0.8 & 0.13 & 0.13 & 0.5 & 0.37 & 0.3 \\
\hline BDT Tropical & 0.8 & 0.27 & 0.45 & 0.8 & 0.1 & 0.1 & 0.5 & 0.3 & 0.3 \\
\hline BDT Temperate & 0.8 & 0.27 & 0.45 & 0.8 & 0.1 & 0.1 & 0.5 & 0.3 & 0.3 \\
\hline BDT Boreal & 0.8 & 0.27 & 0.45 & 0.8 & 0.13 & 0.13 & 0.5 & 0.37 & 0.3 \\
\hline BES Temperate & 0.8 & 0.35 & 0.55 & 0.8 & 0.17 & 0.17 & 0.6 & 0.43 & 0.35 \\
\hline BDS Temperate & 0.8 & 0.35 & 0.55 & 0.8 & 0.17 & 0.17 & 0.6 & 0.43 & 0.35 \\
\hline BDS Boreal & 0.8 & 0.35 & 0.55 & 0.8 & 0.17 & 0.17 & 0.6 & 0.43 & 0.35 \\
\hline C3 Arctic grass & 0.8 & 0.8 & 0.8 & 0.8 & 0.2 & 0.2 & 0.8 & 0.6 & 0.45 \\
\hline $\mathrm{C} 3$ grass & 0.8 & 0.8 & 0.8 & 0.8 & 0.2 & 0.2 & 0.8 & 0.6 & 0.45 \\
\hline $\mathrm{C} 4$ grass & 0.8 & 0.8 & 0.8 & 0.8 & 0.2 & 0.2 & 0.8 & 0.6 & 0.45 \\
\hline Crop1 & 0.8 & 0.8 & 0.8 & 0.8 & 0.2 & 0.2 & 0.8 & 0.6 & 0.45 \\
\hline Crop2 & 0.8 & 0.8 & 0.8 & 0.8 & 0.2 & 0.2 & 0.8 & 0.6 & 0.45 \\
\hline
\end{tabular}

Table S18. PFT-specific information for CTEM.

\begin{tabular}{|c|c|c|c|c|c|c|c|c|}
\hline Plant functional type & $\widehat{R O S_{\max }}$ & $\widehat{F C l, l e a t}$ & $\widehat{F C_{l, s t e m}}$ & FC $\overline{C_{d, l i t t e n}}$ & $\widehat{F K_{\text {leaf }}}$ & & FKstem & FK, root \\
\hline NDL-EVG & $0.54 \times \frac{1}{3.6}$ & 0.42 & 0.12 & 0.30 & 0.06 & & 0.15 & 0.03 \\
\hline NDL-DCD & $0.54 \times \frac{1}{3.6}$ & 0.42 & 0.12 & 0.30 & 0.06 & & 0.15 & 0.03 \\
\hline BDL-EVG & $0.40 \times \frac{1}{3.6}$ & 0.42 & 0.12 & 0.36 & 0.06 & & 0.15 & 0.03 \\
\hline BDL-DCD-COLD & $0.40 \times \frac{1}{3.6}$ & 0.42 & 0.06 & 0.36 & 0.06 & & 0.15 & 0.03 \\
\hline BDL-DCD-DRY & $0.40 \times \frac{1}{3.6}$ & 0.42 & 0.06 & 0.36 & 0.06 & & 0.15 & 0.03 \\
\hline C3 grass & $0.72 \times \frac{1}{3.6}$ & $\begin{cases}0.48 & \text { if green } \\
0.54 & \text { if brown }\end{cases}$ & $\mathrm{n} / \mathrm{a}$ & 0.42 & $\begin{array}{l}0.03 \\
0.02\end{array}$ & $\begin{array}{l}\text { if green } \\
\text { if brown }\end{array}$ & $\mathrm{n} / \mathrm{a}$ & 0.08 \\
\hline $\mathrm{C} 4$ grass & $0.72 \times \frac{1}{3.6}$ & $\begin{cases}0.48 & \text { if green } \\
0.54 & \text { if brown }\end{cases}$ & $\mathrm{n} / \mathrm{a}$ & 0.42 & $\left\{\begin{array}{l}0.03 \\
0.02\end{array}\right.$ & $\begin{array}{l}\text { if green } \\
\text { if brown }\end{array}$ & $\mathrm{n} / \mathrm{a}$ & 0.08 \\
\hline
\end{tabular}


Table S19. PFT-specific information for LM3-FINAL*.

\begin{tabular}{|c|c|c|c|c|c|c|c|}
\hline PFT & $\widehat{F C \text { l, leaf }}$ & $\widehat{F C_{l, s t e m}}$ & $\widehat{F C \widehat{C_{d, l i t t e n}}}$ & $\widehat{F K_{\text {lea }}}$ & $\widehat{F K_{\text {stem }}}$ & $\widehat{F K_{\text {root }}}$ & $\widehat{R O S_{\max }}$ \\
\hline $\mathrm{C} 4$ grass & 0.85 & 1.0 & 0.85 & 0.85 & 0.0 & 0.20 & 0.4 \\
\hline C3 grass & 0.85 & 1.0 & 0.85 & 0.85 & 0.0 & 0.20 & 0.4 \\
\hline Temperate deciduous tree & 0.70 & 0.10 & 0.45 & 0.70 & 0.55 & 0.07 & 0.22 \\
\hline Tropical evergreen tree & 0.70 & 0.15 & 0.50 & 0.70 & 0.60 & 0.10 & 0.22 \\
\hline Boreal evergreen tree & 0.75 & 0.20 & 0.55 & 0.75 & 0.65 & 0.13 & 0.3 \\
\hline
\end{tabular}

Table S20. PFT-specific information for JSBACH-SPITFIRE.

\begin{tabular}{|c|c|c|c|c|c|c|c|c|}
\hline Plant functional type & $\widehat{\widehat{\omega_{*}^{*}}}$ & $\widehat{p b}$ & 固 & $\widehat{C L_{f}}$ & $\widehat{\text { parn }_{1}}$ & $\widehat{\text { para }_{2}}$ & $r_{C A}$ & $p$ \\
\hline Tropical evergreen tree & 0.2 & 25 & 0.1487 & 0.33 & 0.0301 & 0.0281 & 1 & 3 \\
\hline Tropical deciduous tree & 0.3 & 25 & 0.061 & 0.1 & 0.1085 & 0.212 & 0.05 & 3 \\
\hline Extratropical evergreen tree & 0.3 & 20 & 0.1 & 0.33 & 0.0367 & 0.0592 & 1 & 3.75 \\
\hline Extratropical deciduous tree & 0.3 & 22 & 0.371 & 0.33 & 0.0347 & 0.1086 & 1 & 3 \\
\hline Raingreen shrub & 0.3 & 5 & 0.094 & 0.8 & 0.1085 & 0.212 & 1 & 3 \\
\hline Deciduous shrub & 0.3 & 5 & 0.094 & 0.8 & 0.0347 & 0.1086 & 1 & 3 \\
\hline $\mathrm{C} 3$ grass & 0.2 & 2 & $\mathrm{n} / \mathrm{a}$ & $\mathrm{n} / \mathrm{a}$ & $\mathrm{n} / \mathrm{a}$ & & $\mathrm{n} / \mathrm{a}$ & $\mathrm{n} / \mathrm{a}$ \\
\hline $\mathrm{C} 4$ grass & 0.2 & 2 & $\mathrm{n} / \mathrm{a}$ & $\mathrm{n} / \mathrm{a}$ & $\mathrm{n} / \mathrm{a}$ & & $\mathrm{n} / \mathrm{a}$ & $\mathrm{n} / \mathrm{a}$ \\
\hline $\mathrm{C} 3$ pasture & 0.2 & 4 & $\mathrm{n} / \mathrm{a}$ & $\mathrm{n} / \mathrm{a}$ & $\mathrm{n} / \mathrm{a}$ & & $\mathrm{n} / \mathrm{a}$ & $\mathrm{n} / \mathrm{a}$ \\
\hline $\mathrm{C} 4$ pasture & 0.2 & 4 & $\mathrm{n} / \mathrm{a}$ & $\mathrm{n} / \mathrm{a}$ & $\mathrm{n} / \mathrm{a}$ & & $\mathrm{n} / \mathrm{a}$ & $\mathrm{n} / \mathrm{a}$ \\
\hline
\end{tabular}

Table S21. PFT-specific information for JULES-INFERNO. $\widehat{F C}_{\min }$ and $\widehat{F C}_{\max }$ refer to the lower and upper bounds of fuel combustion completeness, with the $l$, leaf and $l$, stem subscripts referring to the live leaf and stem biomass pools, respectively (Table $\mathbf{S 1 2}$ ). Note that all stem biomass is considered subject to combustion - i.e., there is no distinction made between above- and below-ground stem.

\begin{tabular}{|c|c|c|c|c|c|c|}
\hline Plant functional type & $F C_{l, l e a f, \min }$ & $F C_{l, \text { stem, } \max }$ & $F C_{l, \text { stem,min }}$ & $F C_{l, \text { stem,max }}$ & $\widehat{B A_{p} A}$ & $D R$ \\
\hline Broadleaf evergreen tree (tropical) & 0.8 & 1.0 & 0.0 & 0.4 & 0.6 & 0.25 \\
\hline Broadleaf evergreen tree (temperate) & 0.8 & 1.0 & 0.0 & 0.4 & 0.6 & 0.25 \\
\hline Broadleaf deciduous tree & 0.8 & 1.0 & 0.0 & 0.4 & 0.6 & 0.25 \\
\hline Needleleaf evergreen tree & 0.8 & 1.0 & 0.0 & 0.4 & 0.6 & 0.25 \\
\hline Needleleaf deciduous tree & 0.8 & 1.0 & 0.0 & 0.4 & 0.6 & 0.25 \\
\hline $\mathrm{C} 3$ grass & 0.8 & 1.0 & 0.2 & 0.4 & 1.4 & 0.67 \\
\hline $\mathrm{C} 4$ grass & 0.8 & 1.0 & 0.2 & 0.4 & 1.4 & 0.67 \\
\hline Evergreen shrub & 0.8 & 1.0 & 0.2 & 0.4 & 1.2 & 0.33 \\
\hline Deciduous shrub & 0.8 & 1.0 & 0.2 & 0.4 & 1.2 & 0.33 \\
\hline
\end{tabular}


Table S22. Biome-specific information for mortality in LPJ-GUESS-SIMFIRE-BLAZE. $h t_{w, \text { min }}=3.7 \times\left(1-\exp \left[-0.19 \times\left[I_{s u r}\right]\right)\right.$.

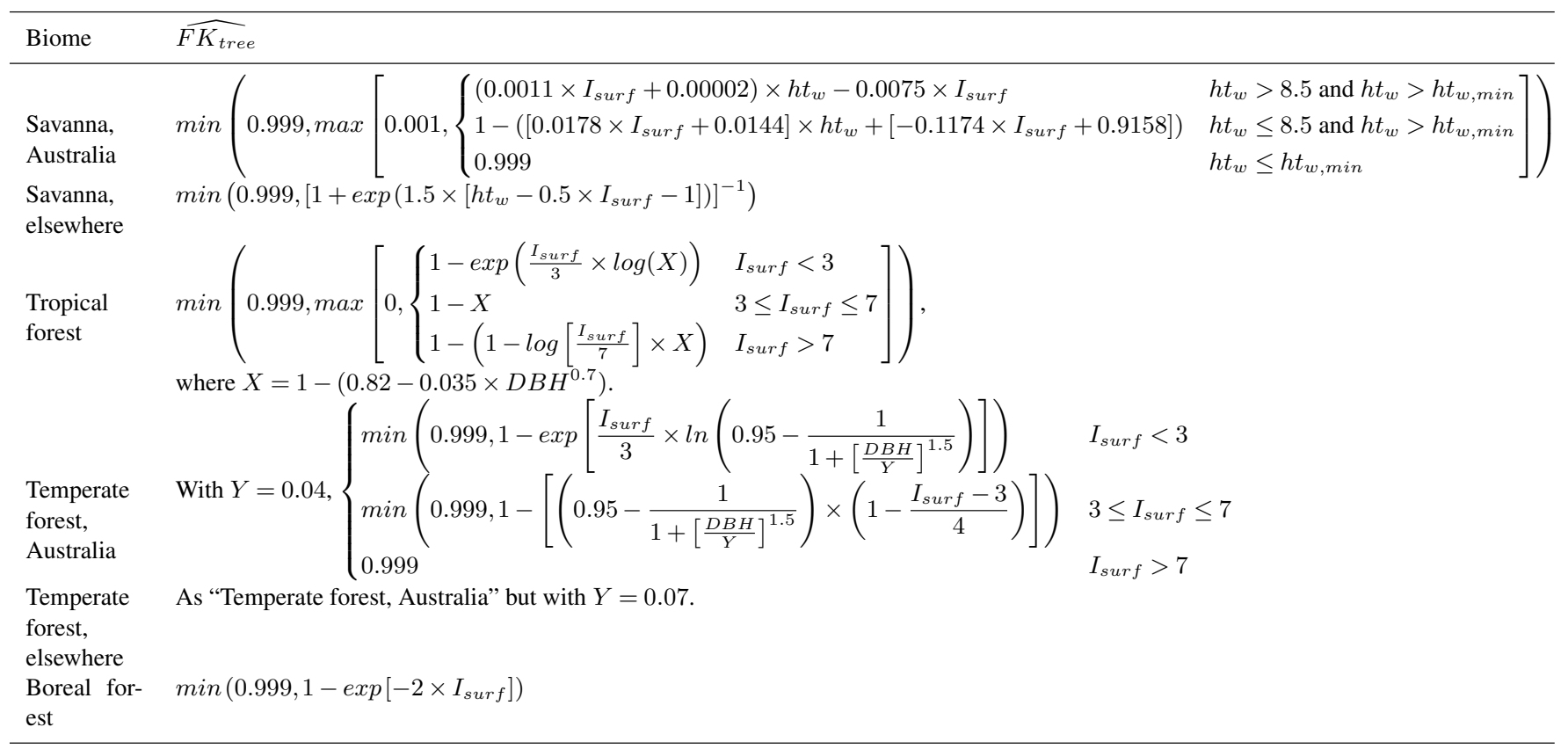

Table S23. PFT-specific information for burned area parameter in LPJ-GUESS-SIMFIRE-BLAZE.

\begin{tabular}{ll}
\hline PFT & a \\
\hline Needleleaved forest & 0.095 \\
Broadleaved forest & 0.092 \\
Mixed forest & 0.127 \\
Shrubland & 0.470 \\
Savanna/grassland & 0.889 \\
Tundra & 0.059 \\
Barren/sparsely vegetated & 0.113 \\
\hline
\end{tabular}


Table S24. PFT-specific information for LPJ-GUESS-GlobFIRM.

\begin{tabular}{lll}
\hline Vegetation type & 目 & $\widehat{\theta}$ \\
\hline Boreal needleleaved tree & 0.3 & 0.3 \\
Temperate needleleaved evergreen tree & 0.3 & 0.3 \\
Temperate broadleaved deciduous tree & 0.1 & 0.3 \\
Temperate broadleaved evergreen tree & 0.3 & 0.3 \\
Tropical broadleaved evergreen tree & 0.1 & 0.3 \\
Tropical broadleaved raingreen tree & 0.3 & 0.3 \\
Grass & 0.5 & 0.2 \\
\hline
\end{tabular}

Table S25. PFT-specific information for LPJ-GUESS-SPITFIRE. Fuel bulk density for tropical tree PFTs $\rho_{b, t r o p .}=\max (2,15.84 \times$ $\left.\exp \left[-85 \times L_{d+l, g}\right]+2.22 \times \exp \left[-2.045 \times L_{d+l, g}\right]\right)$.

\begin{tabular}{|c|c|c|c|c|c|c|c|c|c|c|}
\hline Plant functional type & $k_{2}$ & $k_{3}$ & $\widehat{B D T}$ & $\overline{C L}$ & $\begin{array}{l}\text { Max } \\
\text { crown } \\
\text { area }\end{array}$ & $\widehat{\mathrm{DU}}$ & $\widehat{\omega_{*}}$ & F & $r C A$ & р \\
\hline Boreal needleleaved evergreen tree & 60 & 0.67 & $0.0292 \times \overline{D B H}+0.2632$ & 0.33 & 50 & 25 & 0.3 & 0.11 & 1 & 3 \\
\hline $\begin{array}{l}\text { Boreal shade-intolerant needleleaved ever- } \\
\text { green tree }\end{array}$ & 60 & 0.67 & $0.0292 \times \overline{D B H}+0.2633$ & 0.33 & 50 & 25 & 0.3 & 0.11 & 1 & 3 \\
\hline Boreal needleleaved summergreen tree & 60 & 0.67 & $0.0347 \times D B H+0.1086$ & 0.33 & 50 & 25 & 0.3 & 0.094 & 1 & 3 \\
\hline $\begin{array}{l}\text { Boreal shade-intolerant broadleaved sum- } \\
\text { mergreen tree }\end{array}$ & 60 & 0.67 & $0.0347 \times \overline{D B H}+0.1086$ & 0.33 & 50 & 22 & 0.3 & 0.094 & 1 & 3 \\
\hline $\begin{array}{l}\text { Temperate shade-intolerant broadleaved } \\
\text { summergreen tree }\end{array}$ & 60 & 0.67 & $0.0347 \times \mathrm{DBH}+0.1086$ & 0.33 & 50 & 22 & 0.3 & 0.094 & 1 & 3 \\
\hline Temperate broadleaved summergreen tree & 60 & 0.67 & $0.0347 \times D B H+0.1087$ & 0.33 & 50 & 22 & 0.3 & 0.094 & 1 & 3 \\
\hline Temperate broadleaved evergreen tree & 60 & 0.67 & $0.0451 \times \overline{D B H}+0.1412$ & 0.33 & 50 & 10 & 0.3 & 0.371 & 0.95 & 3 \\
\hline Temperate needleleaved evergreen tree & 60 & 0.67 & $0.0367 \times \mathrm{DBH}+0.0592$ & 0.33 & 50 & 25 & 0.3 & 0.1 & 1 & 3.75 \\
\hline Tropical broadleaved evergreen tree & 39 & 0.53 & $15.95-14.23 \times 0.98456^{\text {DBता }}$ & 0.33 & 300 & $\rho_{b, \text { trop }}$ & 0.2 & 0.1487 & 1 & 3 \\
\hline $\begin{array}{l}\text { Tropical shade-intolerant broadleaved ever- } \\
\text { green tree }\end{array}$ & 39 & 0.53 & $15.95-14.23 \times 0.98456^{\text {एकास }}$ & 0.33 & 300 & $\rho_{b, \text { trop. }}$ & 0.2 & 0.1487 & 1 & 3 \\
\hline Tropical broadleaved raingreen tree & 13.18 & 0.61 & 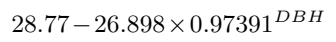 & 0.1 & 300 & $\rho_{b, \text { trop }}$ & 0.3 & 0.061 & 0.05 & 3 \\
\hline Cool/temperate $(\mathrm{C} 3)$ grass & $\mathrm{n} / \mathrm{a}$ & $\mathrm{n} / \mathrm{a}$ & $\mathrm{n} / \mathrm{a}$ & $\mathrm{n} / \mathrm{a}$ & $\mathrm{n} / \mathrm{a}$ & 2 & 0.2 & 0.05 & 0 & 0 \\
\hline Warm $(\mathrm{C} 4)$ grass & $\mathrm{n} / \mathrm{a}$ & $\mathrm{n} / \mathrm{a}$ & $\mathrm{n} / \mathrm{a}$ & $\mathrm{n} / \mathrm{a}$ & $\mathrm{n} / \mathrm{a}$ & 2 & 0.2 & 0.05 & 0 & 0 \\
\hline
\end{tabular}


Table S26. PFT-specific information for LPJ-LMfire: $\widehat{\underline{L_{L}} i}$ is PFT-specific lightning ignition efficiency, and $\widehat{\omega_{\sharp} l}$ is PFT-specific moisture of extinction for live fuels.

\begin{tabular}{|c|c|c|c|c|c|c|c|c|}
\hline PFT & $\widehat{\overline{L E}_{i}}$ & $\widehat{\omega * \vartheta} \iota$ & $\widehat{C L}$ & par1 & $\widehat{\text { para }_{2}}$ & 同 & $D_{1}$ & $D_{2}$ \\
\hline Tropical broadleaved evergreen tree & 0.05 & 0.2 & $1 / 3$ & 0.0301 & 0.0281 & 0.16 & 1.75 & -3.75 \\
\hline Tropical broadleaved raingreen tree & 0.4 & 0.3 & 0.1 & 0.1085 & 0.2120 & 0.351 & 1.90 & -4.20 \\
\hline Temperate needleleaved evergreen tree & 0.1 & 0.3 & $1 / 3$ & 0.0670 & 0.5590 & 0.094 & 2.57 & -6.20 \\
\hline Temperate broadleaved evergreen tree & 0.1 & 0.3 & $1 / 3$ & 0.0451 & 0.1412 & 0.07 & 1.23 & -2.20 \\
\hline Temperate broadleaved summergreen tree & 0.5 & 0.3 & $1 / 3$ & 0.0347 & 0.1086 & 0.094 & 1.68 & -3.53 \\
\hline Boreal needleleaved evergreen tree & 0.44 & 0.35 & $1 / 3$ & 0.0292 & 0.1086 & 0.094 & 1.23 & -2.20 \\
\hline Boreal summergreen tree & 0.44 & 0.35 & $1 / 3$ & 0.0347 & 0.1086 & 0.094 & 1.32 & -2.46 \\
\hline C3 perennial grass & 0.5 & 0.2 & $\mathrm{n} / \mathrm{a}$ & $\mathrm{n} / \mathrm{a}$ & $\mathrm{n} / \mathrm{a}$ & $\mathrm{n} / \mathrm{a}$ & $\mathrm{n} / \mathrm{a}$ & $\mathrm{n} / \mathrm{a}$ \\
\hline $\mathrm{C} 4$ perennial grass & 0.5 & 0.2 & $\mathrm{n} / \mathrm{a}$ & $\mathrm{n} / \mathrm{a}$ & $\mathrm{n} / \mathrm{a}$ & $\mathrm{n} / \mathrm{a}$ & $\mathrm{n} / \mathrm{a}$ & $\mathrm{n} / \mathrm{a}$ \\
\hline
\end{tabular}




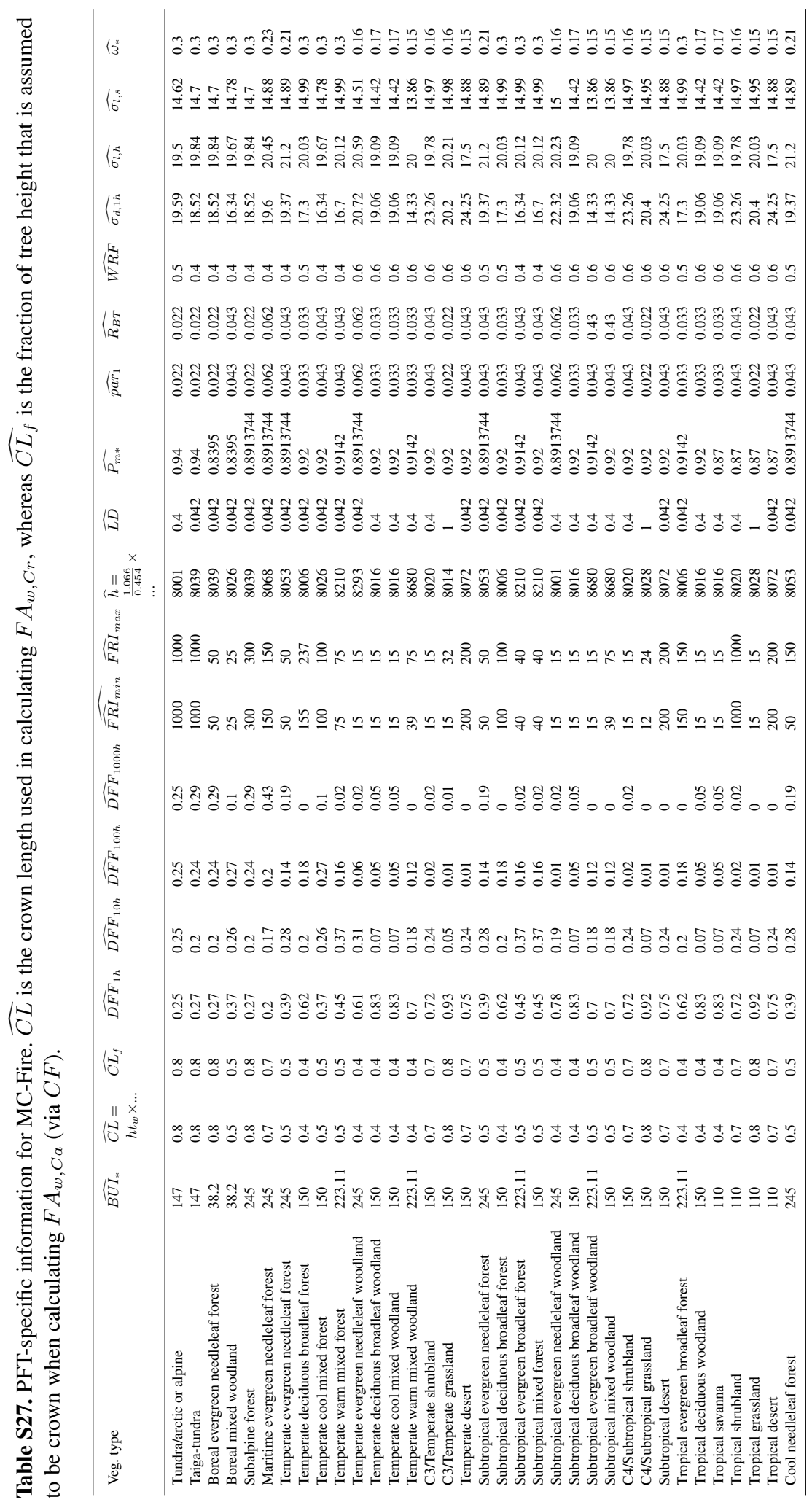


Table S28. PFT-specific information for ORCHIDEE-SPITFIRE. ORCHIDEE is capable of simulating woody vegetation height, but height

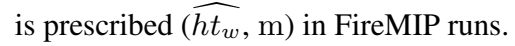

\begin{tabular}{|c|c|c|c|c|c|c|c|c|c|c|c|}
\hline Plant functional type & $\widehat{\underline{\theta \theta}}$ & $\widehat{h t_{n}}$ & $\overline{C L}$ & par & aar, & $\mathrm{rCt}$ & D & F & $\widehat{\mathrm{W}}$ & $F C_{d, 100 h, \max }$ & $F \bar{C}_{d, 1000 h, \max }$ \\
\hline Tropical broadleaf evergreen trees & 25 & 50 & 0.33 & 0.03 & 0.03 & 0.95 & 3 & 0.15 & 0.2 & 0.65 & 0.41 \\
\hline Tropical broadleaf raingreen trees & 25 & 50 & 0.1 & 0.11 & 0.21 & 0.05 & 3 & 0.06 & 0.3 & 0.65 & 0.41 \\
\hline Temperate needleleaf evergreen trees & 25 & 30 & 0.33 & 0.07 & 0.56 & 0.95 & 3.75 & 0.1 & 0.3 & 0.73 & 0.38 \\
\hline Temperate broadleaf evergreen trees & 10 & 30 & 0.33 & 0.05 & 0.14 & 0.95 & 3 & 0.37 & 0.3 & 0.73 & 0.38 \\
\hline Temperate broadleaf summergreen trees & 22 & 30 & 0.33 & 0.04 & 0.11 & 0.95 & 3 & 0.09 & 0.3 & 0.73 & 0.38 \\
\hline Boreal needleleaf evergreen trees & 25 & 20 & 0.67 & 0.03 & 0.26 & 0.95 & 3 & 0.11 & 0.35 & 0.73 & 0.38 \\
\hline Boreal broadleaf summergreen trees & 22 & 20 & 0.33 & 0.04 & 0.11 & 0.95 & 3 & 0.09 & 0.35 & 0.73 & 0.38 \\
\hline Boreal needleleaf summergreen trees & 22 & 20 & 0.33 & 0.04 & 0.11 & 0.95 & 3 & 0.09 & 0.35 & 0.73 & 0.38 \\
\hline $\mathrm{C} 3$ grass & 2 & 0.2 & $\mathrm{n} / \mathrm{a}$ & $\mathrm{n} / \mathrm{a}$ & $\mathrm{n} / \mathrm{a}$ & $\mathrm{n} / \mathrm{a}$ & $\mathrm{n} / \mathrm{a}$ & $\mathrm{n} / \mathrm{a}$ & 0.2 & 0.76 & 0.76 \\
\hline $\mathrm{C} 4$ grass & 2 & 0.2 & $\mathrm{n} / \mathrm{a}$ & $\mathrm{n} / \mathrm{a}$ & $\mathrm{n} / \mathrm{a}$ & $\mathrm{n} / \mathrm{a}$ & $\mathrm{n} / \mathrm{a}$ & $\mathrm{n} / \mathrm{a}$ & 0.2 & 0.76 & 0.76 \\
\hline $\mathrm{C} 3$ agriculture & 2 & 0.4 & $\mathrm{n} / \mathrm{a}$ & $\mathrm{n} / \mathrm{a}$ & $\mathrm{n} / \mathrm{a}$ & $\mathrm{n} / \mathrm{a}$ & $\mathrm{n} / \mathrm{a}$ & $\mathrm{n} / \mathrm{a}$ & 0.2 & 0.35 & 0.35 \\
\hline $\mathrm{C} 4$ agriculture & 2 & 0.4 & $\mathrm{n} / \mathrm{a}$ & $\mathrm{n} / \mathrm{a}$ & $\mathrm{n} / \mathrm{a}$ & $\mathrm{n} / \mathrm{a}$ & $\mathrm{n} / \mathrm{a}$ & $\mathrm{n} / \mathrm{a}$ & 0.2 & 0.35 & 0.35 \\
\hline
\end{tabular}



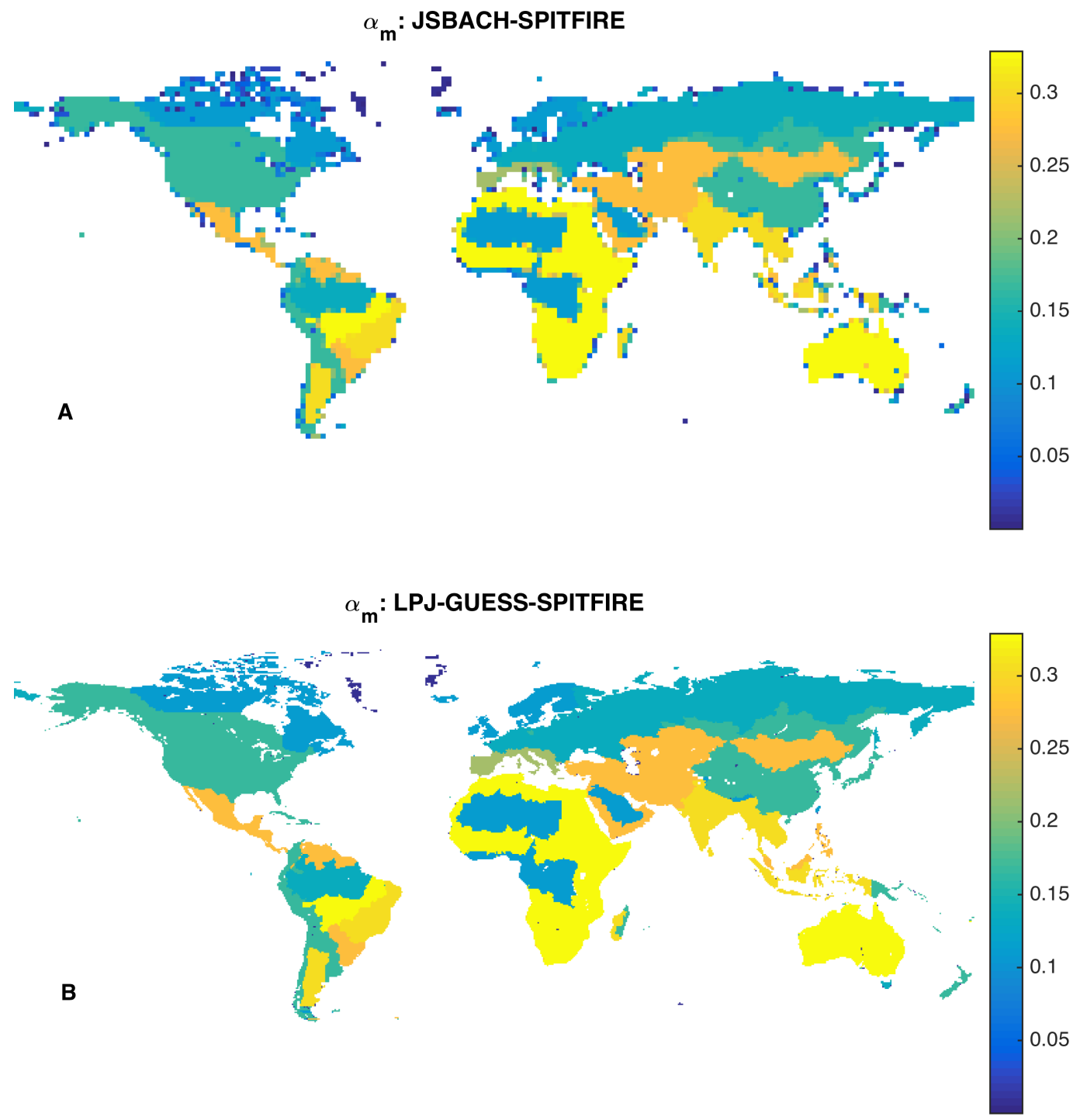

Figure S1. Monthly ignitions per person $\left(\underline{\alpha_{m}}\right)$ for (a) JSBACH-SPITFIRE and (b) LPJ-GUESS-SPITFIRE. See Table $\mathbf{S ]}$ for usage details. 


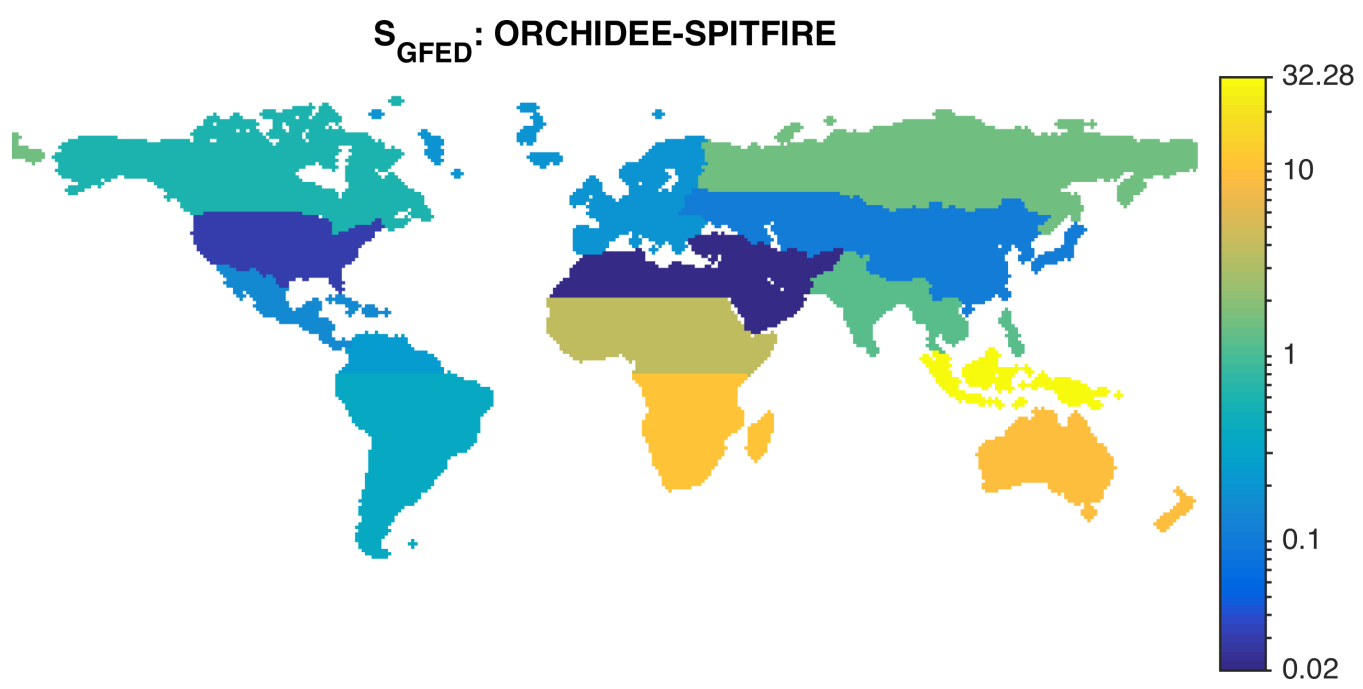

Figure S2. Scaling factor $S_{G F E D}$ (Table S4) for ORCHIDEE-SPITFIRE. Note logarithmic color scale. This map is based on the GFED regions (van der Wert et al, 2006) and does not exactly match the mask used by ORCHIDEE-SPITFIRE. 


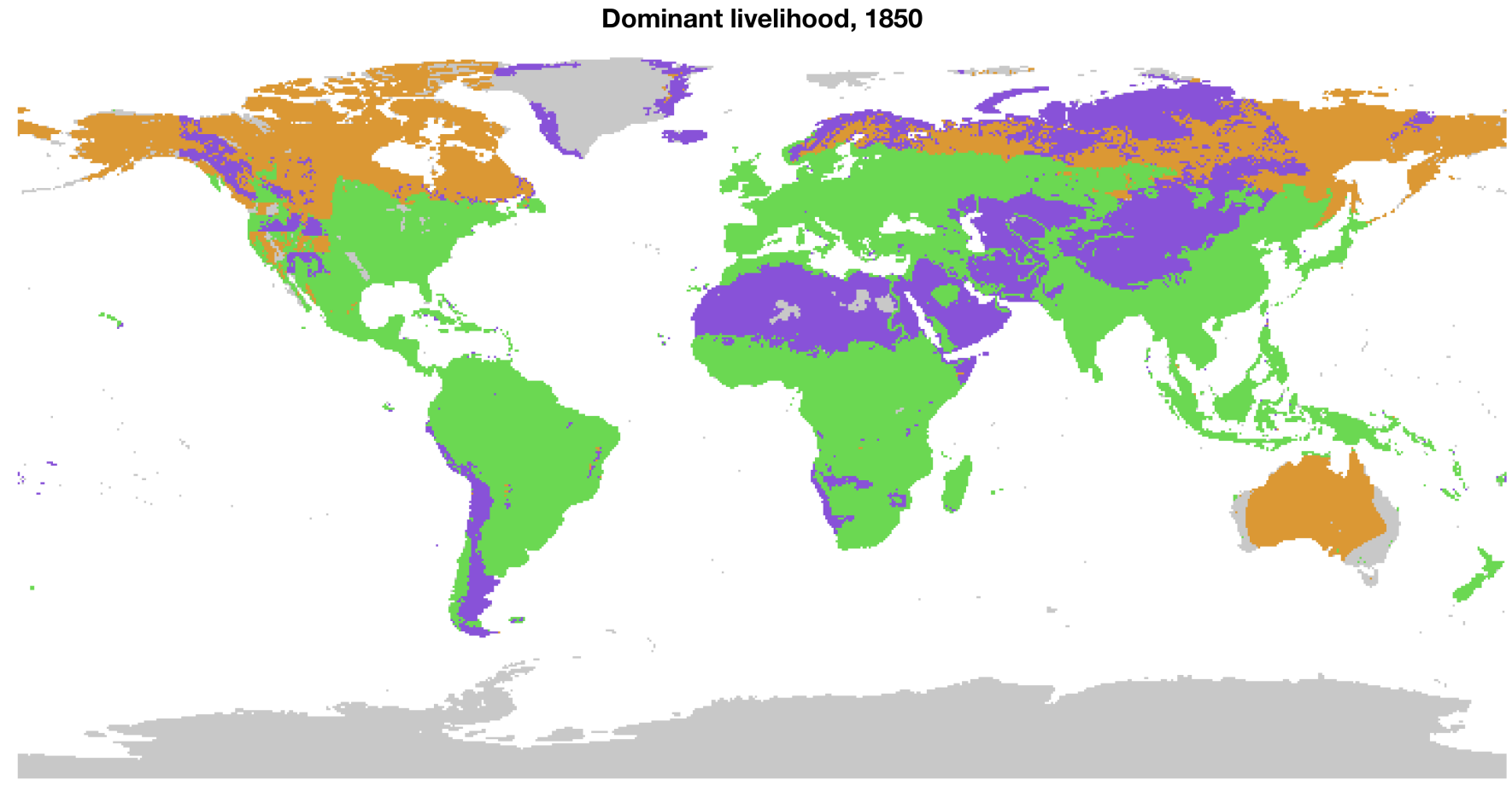

Farmers

Pastoralists

Hunter-gatherers

No fire-starters

Figure S3. Dominant livelihoods used by LPJ-LMfire for 1850 onwards. Note that this is only used for calculating human ignitions (Table SII). 


\section{References}

Best, M. J., Pryor, M., Clark, D. B., Rooney, G. G., Essery, R. L. H., Ménard, C. B., Edwards, J. M., Hendry, M. A., Porson, A., Gedney, N., Mercado, L. M., Sitch, S., Blyth, E., Boucher, O., Cox, P. M., Grimmond, C. S. B., and Harding, R. J.: The Joint UK Land Environment Simulator (JULES), model description - Part 1: Energy and water fluxes, Geoscientific Model Development, 4, 677-699, doi:10.5194/gmd-4-677-2011, http://www.geosci-model-dev.net/4/677/2011/, 2011.

Giglio, L., Randerson, J. T., van der Werf, G. R., Kasibhatla, P., Collatz, G., Morton, D. C., and DeFries, R.: Assessing variability and longterm trends in burned area by merging multiple satellite fire products, Biogeosciences, 7, 1171-1186, www.biogeosciences.net/7/1171/ 2010)/, 2010.

Goff, J. A. and Gratch, S.: Low-pressure properties of water from -160 to $212^{\circ} \mathrm{F}$, Transactions of the American Society of Heating and Ventilating Engineers, pp. 95-122, 1946.

Keetch, J. J. and Byram, G. M.: A Drought Index for Forest Fire Control, Tech. Rep. USDA For. Serv. Res. Paper SE-38, http://www. treesearch.ts.ted.us/pubs/40, 1968.

Knorr, W., Kaminski, T., Arneth, A., and Weber, U.: Impact of human population density on fire frequency at the global scale, Biogeosciences, 11, 1085-1102, doi:10.5194/bg-11-1085-2014, http://www.biogeosciences.net/11/1085/2014/, 2014.

15 Melton, J. R. and Arora, V. K.: Competition between plant functional types in the Canadian Terrestrial Ecosystem Model (CTEM) v. 2.0, Geoscientific Model Development, 9, 323-361, doi:I0.5194/gmd-9-323-2016, 2016.

Nesterov, V.: Forest fires and methods of fire risk determination, Goslesbumizdat, Moscow, 1949.

Noble, I. R., Gill, A. M., and Bary, G. A. V.: McArthur's fire-danger meters expressed as equations, Australian Journal of Ecology, 5, 201-203, doi:10.1111/j.1442-9993.1980.tb01243.x, http://doi.wiley.com/10.1111/j.1442-9993.1980.tb01243.x, 1980.

20 Rabin, S. S., Magi, B. I., Shevliakova, E., and Pacala, S. W.: Quantifying regional, time-varying effects of cropland and pasture on vegetation fire, Biogeosciences, 12, 6591-6604, doi:10.5194/bg-12-6591-2015, http://www.biogeosciences.net/12/6591/2015/, 2015.

Sitch, S., Smith, B., Prentice, I. C., Arneth, A., Bondeau, A., Cramer, W., Kaplan, J. O., Levis, S., Lucht, W., Sykes, M. T., Thonicke, K., and Venevsky, S.: Evaluation of ecosystem dynamics, plant geography and terrestrial carbon cycling in the LPJ dynamic global vegetation model, Global Change Biology, 9, 161-185, doi:10.1046/j.1365-2486.2003.00569.x, http://onlinelibrary.wiley.com/doi/10.1046/j. 1365-2486.20(03.0(0569.x/abstract, 2003.

Thonicke, K., Spessa, A., Prentice, I. C., Harrison, S. P., Dong, L., and Carmona-Moreno, C.: The influence of vegetation, fire spread and fire behaviour on biomass burning and trace gas emissions: results from a process-based model, Biogeosciences, 7, 1991-2011, doi:10.5194/bg-7-1991-2010, http://www.biogeosciences.net/7/1991/2010/bg-7-1991-2010.html, 2010.

van der Werf, G. R., Randerson, J. T., Giglio, L., Collatz, G. J., Kasibhatla, P. S., and Arellano Jr., A. F.: Interannual variability in global biomass burning emissions from 1997 to 2004, Atmospheric Chemistry and Physics, 6, 3423-3441, doi:10.5194/acp-6-3423-2006, http: //www.atmos-chem-phys.net/6/3423/2006/, 2006.

van der Werf, G. R., Randerson, J. T., Giglio, L., Collatz, G. J., Mu, M., Kasibhatla, P. S., Morton, D. C., Defries, R. S., Jin, Y., and van Leeuwen, T. T.: Global fire emissions and the contribution of deforestation, savanna, forest, agricultural, and peat fires (1997-2009), Atmospheric Chemistry and Physics, 10, 11 707-11 735, doi:10.5194/acp-10-11707-2010, http://www.atmos-chem-phys.net/10/11707/ 35 2(1)/, 2010.

van Wagner, C. E.: Development and Structure of the Canadian Forest Fire Weather Index System, Tech. Rep. For. Tech. Rep. 35, Ottawa, ON, 1987.

van Wagner, C. E. and Pickett, T. L.: Equations and FORTRAN Program for the Canadian Forest Fire Weather Index System, Tech. Rep. Forestry Technical Report 33, Ottawa, 1985. 